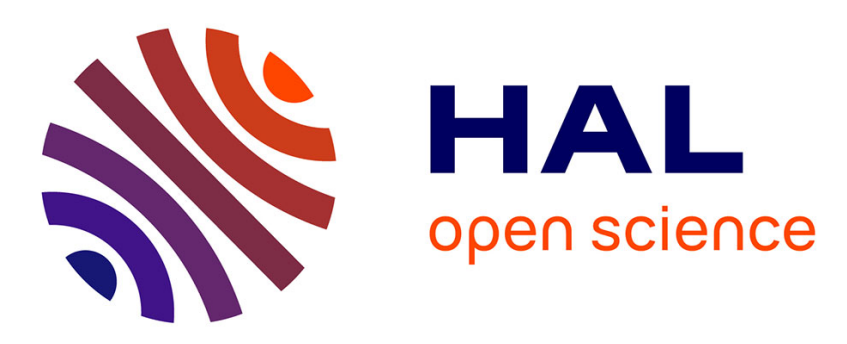

\title{
Aggregating Tastes, Beliefs, and Attitudes under Uncertainty
}

Eric Danan, Thibault Gajdos, Brian Hill, Jean-Marc Tallon

\section{To cite this version:}

Eric Danan, Thibault Gajdos, Brian Hill, Jean-Marc Tallon. Aggregating Tastes, Beliefs, and Attitudes under Uncertainty. 2014. halshs-01099032

\section{HAL Id: halshs-01099032 https://shs.hal.science/halshs-01099032}

Submitted on 30 Dec 2014

HAL is a multi-disciplinary open access archive for the deposit and dissemination of scientific research documents, whether they are published or not. The documents may come from teaching and research institutions in France or abroad, or from public or private research centers.
L'archive ouverte pluridisciplinaire HAL, est destinée au dépôt et à la diffusion de documents scientifiques de niveau recherche, publiés ou non, émanant des établissements d'enseignement et de recherche français ou étrangers, des laboratoires publics ou privés. 


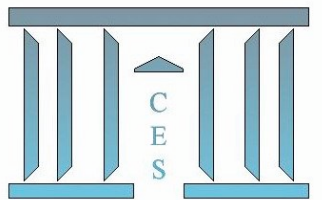

Aggregating Tastes, Beliefs, and Attitudes under Uncertainty

Eric DANAN, Thibault GAJDOS, Brian HILL, Jean-Marc TALLON

2014.63

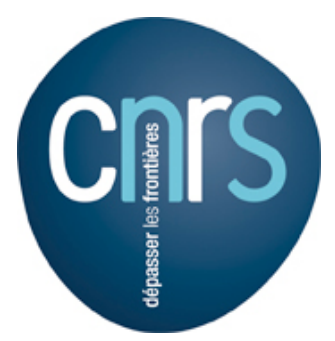




\title{
Aggregating Tastes, Beliefs, and Attitudes under Uncertainty*
}

\author{
Eric Danan ${ }^{\dagger} \quad$ Thibault Gajdos ${ }^{\ddagger} \quad$ Brian Hill Sean-Marc Tallon $^{\S}$
}

July 20, 2014

\begin{abstract}
We provide possibility results on the aggregation of beliefs and tastes for Monotone, Bernoullian and Archimedian preferences of Cerreia-Vioglio, Ghirardato, Maccheroni, Marinacci, and Siniscalchi (2011). We propose a new axiom, Unambiguous Pareto Dominance, which requires that if the unambiguous part of individuals' preferences over a pair of acts agree, then society should follow them. We characterize the resulting social preferences and show that it is enough that individuals share a prior to allow non dictatorial aggregation. A further weakening of this axiom on common-taste acts, where cardinal preferences are identical, is also characterized. It gives rise to a set of relevant priors at the social level that can be any subset of the convex hull of the individuals' sets of relevant priors. We then apply these general results to the Maxmin Expected Utility model, the Choquet Expected Utility model and the Smooth Ambiguity model. We end with a characterization of the aggregation of ambiguity attitudes.
\end{abstract}

Keywords. Preference Aggregation, Social Choice, Uncertainty

JEL Classification. D71, D81

\section{Introduction}

Many social decisions have to be made in uncertain environments where individuals have both different tastes over the possible outcomes and different beliefs over the possible states of the world. As Hylland and Zeckhauser (1979) and Mongin (1995, 1998) have showed, for Subjective Expected Utility (SEU) preferences (Savage, 1954; Anscombe and Aumann, 1963), simultaneous

\footnotetext{
${ }^{*}$ We thank Tzachi Gilboa, Peter Klibanoff, Philippe Mongin, Sujoy Mukerji, Klaus Nehring, Efe Ok, Marcus Pivato, Xiangyu Qu, David Schmeidler, Peter Wakker, and Stéphane Zuber, as well as participants to the D-TEA 2014 meeting, DRI seminar in Paris, and workshop in Bielefeld University, for useful comments and discussions. Danan thanks support from the Labex MME-DII program (ANR-11-LBX-0023-01). Gajdos thanks support from the $A^{\star}$ MIDEX project (ANR-11-IDEX-0001-02) funded by the Investissements d'Avenir program. Hill thanks support from the Investissements d'Avenir program (ANR-11-IDEX-0003/Labex Ecodec/ANR-11-LABX0047). Tallon thanks support from ANR grant AmGames (ANR-12-FRAL-0008-01) and from the Investissements d'Avenir program (ANR-10-LABX-93).

${ }_{\dagger}^{\dagger}$ THEMA, Université de Cergy-Pontoise, CNRS, 33 boulevard du Port, 95011 Cergy-Pontoise cedex, France (eric.danan@u-cergy.fr).

${ }^{\ddagger}$ Greqam, CNRS, EHESS, Université d'Aix-Marseille (Aix Marseille School of Economics) (thibault.gajdos@univ-amu.fr).

${ }^{\S}$ GREGHEC, CNRS, HEC Paris (hill@hec.fr).

`Paris School of Economics, CNRS (jean-marc.tallon@univ-paris1.fr).
} 
heterogeneity of tastes and beliefs leads to an impossibility of Paretian aggregation of individual preferences into a social preference. Mongin $(1995,1998,2014)$ and Gilboa, Samet, and Schmeidler (2004) have argued that the problem can be traced to spurious unanimity, that is, cases of unanimous preferences that result from differences in beliefs and tastes that "cancel" each other out. Indeed, the possibility of Paretian preference aggregation can be restored by restricting the Pareto principle to cases of identical beliefs. ${ }^{1}$

As famously noted by Ellsberg (1961), individuals may violate Subjective Expected Utility, in particular when they perceive and are sensitive to ambiguity. Individuals are then characterized by their beliefs - captured for instance by a set of probability distributions -, tastes over outcomes, and ambiguity attitudes. As Gajdos, Tallon, and Vergnaud (2008) have shown, this third factor - ambiguity attitudes - exacerbates the difficulties for aggregation: as soon as some individuals are ambiguity sensitive (that is, display a non-neutral ambiguity attitude), Paretian aggregation in the presence of heterogeneous tastes is impossible. Moreover, possibility cannot be restored by restricting the Pareto principle to cases of identical beliefs.

To illustrate the role of commonality of beliefs and of ambiguity attitudes in the aggregation problem, consider a country with an important fishery sector and an influential sector specialized in environmental technologies. If the average water temperature increases by 3 degrees or more, the fish stocks on the country's coastline will be wiped out by predators that survive in warmer water, and the country has to decide whether to invest now in an expensive defense mechanism developed by its technology sector. If the mechanism is installed, it will significantly decrease the normal fish yield if the temperature rise is below 3 degrees, but it will ensure a continued yield if the temperature rise is higher. The technology sector believes that the temperature will only increase by 2 degrees; however, it is in favor of the development, which constitutes an important investment in its product. The fishery sector believes that the temperature increase will be at least 4 degrees; accordingly, it is also in favor of installing the mechanism. Although the Pareto principle recommends installation, there is no unanimity about the reasons behind this decision. In particular, the fishing sector's approval of the installation is based on their beliefs, and if they shared the same beliefs as the technology sector, they would cease to approve of the installation. This is a case of spurious unanimity as described by Mongin (1995, 1998, 2014) and Gilboa, Samet, and Schmeidler (2004), who argue that the Pareto principle is not compelling in these situations.

Now suppose that rather than having precise probabilistic beliefs, the two sectors perceive ambiguity, and have beliefs representable by the same set of probability distributions, allowing for temperature increases ranging from 2 to 4 degrees. If they are ambiguity averse, and form preferences by the Maxmin Expected Utility rule (Gilboa and Schmeidler, 1989), then their preferences will be as in the case just discussed. In particular, the fishery sector will prefer to install the mechanism, because it focuses on the worst-case scenario, which involves extinction of the fish stock if no action is taken. Again, the Pareto principle recommends installation; again, questions may be raised about the quality of this recommendation. Unlike the previous case, it is not the difference in beliefs that is at issue, but the perception of particular options as hedging

\footnotetext{
${ }^{1}$ More precisely, possibility is reinstated when the Pareto principle is restricted to acts (state-contingent consequences) that depend on events over which individuals have the same probabilistic beliefs (Gilboa, Samet, and Schmeidler, 2004).
} 
opportunities: the fishery sector thinks of the installation as hedging the uncertainty about the temperature, and, since the sector is ambiguity averse, prefers it for this reason. Moreover, this renders the judgement particularly sensitive to background risk positions: if the country had signed an "insurance treaty" with a neighbor allowing the home fishermen to fish in their neighbor's safe waters in the eventuality of a significant temperature rise, then the installation would cease to be a hedging option for the fishery sector and it would no longer approve of it.

For ambiguity-sensitive agents, differences in perceptions concerning hedging over ambiguous events can thus be a further source of spurious unanimity, and accordingly a further challenge to the appeal of the Pareto principle. We shall use the term spurious hedging to refer to this phenomenon. The goal of this paper is to identify versions of the Pareto principle that are immune to both spurious unanimity and spurious hedging and thereby restore the possibility of preference aggregation for ambiguity-sensitive decision makers. In order to do this, we first need to disentangle the taste, belief and ambiguity attitude components of preference aggregation. Working in an Anscombe-Aumann setting, we consider the broad class of Monotone, Bernoullian, Archimedean (MBA) preferences (Cerreia-Vioglio, Ghirardato, Maccheroni, Marinacci, and Siniscalchi, 2011), which subsumes many of the popular models of decision under uncertainty. Individuals' beliefs, which correspond to a possibly incomplete unambiguous preference subrelation, consist of sets of probability distributions. An act (state-contingent lottery) is unambiguously preferred to another if it provides a higher expected utility for every prior in the set, as originally modelled by Bewley (2002). As the name suggests, an individual's unambiguous preference is independent of her attitude toward ambiguity, and accordingly is robust to hedging.

Our main axiom - Unambiguous Pareto Dominance - is the restriction of the usual Pareto Dominance axiom to unambiguous preferences: if an act $f$ is unambiguously preferred to an act $g$ by every member of the society, then it should also be unambiguously preferred by society. In other words, if, for each individual, the expected utility of $f$ is higher than the expected utility of $g$ for every prior in her set of beliefs, then society should also have higher expected utility for $f$ than for $g$ under every prior in its set of beliefs. This dominance notion only involves preferences that are robust to hedging, and hence does not yield the spurious unanimity recommendation in the example above. Imposing Unambiguous Pareto Dominance yields a social utility that is a linear aggregation of the individuals' utility functions and a set of social beliefs that is contained in the intersection of the individuals' sets. It restores the possibility that was threatened by the result of Gajdos, Tallon, and Vergnaud (2008), insofar as it allows non-dictatorial aggregation of ambiguity-sensitive preferences, as long as the individuals have compatible beliefs, in the sense that they have at least one prior in common. It does however fall prey to the impossibility identified on SEU preferences by Hylland and Zeckhauser (1979) and Mongin $(1995,1998)$, insofar as in the absence of sufficient consensus among individuals, only dictatorship is compatible with Unambiguous Pareto Dominance.

We thus consider a further weakening of Unambiguous Pareto Dominance to "commontaste acts", in a manner similar to the restriction of Pareto Dominance to common-belief acts by Gilboa, Samet, and Schmeidler (2004). ${ }^{2}$ This fully restores possibility, even in the case

\footnotetext{
${ }^{2}$ Acts are common-taste acts if all individuals have the same cardinal preferences on the (convex hull of the)
} 
of conflicting probabilistic beliefs (disjoint sets of priors). Finally, we introduce a new axiom allowing aggregation of ambiguity attitudes, independently of beliefs and tastes.

The paper is constructed as follows. The setup is presented in Section 2. Section 3 provides the relevant material on MBA preferences. Section 4 states Harsanyi's result in our framework and provides worked-out examples of the sources of the impossibility results mentioned above (spurious unanimity and spurious hedging). The main axioms (Unambiguous Pareto Dominance and its restriction to common-taste acts) and results (aggregation results) are contained in Section 5. We then apply these general results to three classes of preferences: Maxmin Expected Utility, Choquet Expected Utility, and Smooth Ambiguity preferences in Section 6. Section 7 deals with the aggregation of ambiguity attitudes. Section 8 contains a discussion of some related literature. Proofs are gathered in the Appendix.

\section{Setup}

We adopt a simple Anscombe-Aumann setting with a finite set $\mathcal{S}$ of states of the world and a finite set of $\mathcal{X}$ of prizes. Let $\mathcal{P}=\Delta(\mathcal{X})$ denote the set of all (roulette) lotteries and $\mathcal{F}=\mathcal{P}^{\mathcal{S}}$ denote the set of all acts (horse-roulette lotteries). Given two acts $f, g \in \mathcal{F}$ and $\lambda \in[0,1]$, let $\lambda f+(1-\lambda) g \in \mathcal{F}$ denote the act yielding lottery $\lambda f(s)+(1-\lambda) g(s)$ in each state $s \in \mathcal{S}$.

Given a lottery $p \in \mathcal{P}$, we abuse notation by also letting $p \in \mathcal{F}$ denote the corresponding constant act. Given a utility function $u: \mathcal{X} \rightarrow \mathbb{R}$ and a lottery $p \in \mathcal{P}$, let $u \circ p=E_{p}(u(x)) \in \mathbb{R}$, where $E_{p}$ denotes the expectation operator with respect to $p$. Given a utility function $u: \mathcal{X} \rightarrow \mathbb{R}$ and an act $f \in \mathcal{F}$, let $u \circ f=(u \circ(f(s)))_{s \in \mathcal{S}} \in \mathbb{R}^{\mathcal{S}}$.

Given a binary relation $\succsim$ on $\mathcal{F}$, let $\succ$ and $\sim$ denote its asymmetric and symmetric components, respectively. As a benchmark we recall the classical axioms and representation of the Subjective Expected Utility (henceforth SEU) model in the Anscombe-Aumann setting.

Axiom (Completeness). For all $f, g \in \mathcal{F}, f \succsim g$ or $g \succsim f$.

Axiom (Transitivity). For all $f, g, h \in \mathcal{F}$, if $f \succsim g$ and $g \succsim h$ then $f \succsim h$.

Axiom (Non-Triviality). There exist $f, g \in \mathcal{F}$ such that $f \succ g$.

Axiom (Monotonicity). For all $f, g \in \mathcal{F}$, if $f(s) \succsim g(s)$ for all $s \in \mathcal{S}$ then $f \succsim g$.

Axiom (Mixture Continuity). For all $f, g, h \in \mathcal{F}$, the sets $\{\lambda \in[0,1]: \lambda f+(1-\lambda) g \succsim h\}$ and $\{\lambda \in[0,1]: h \succsim \lambda f+(1-\lambda g)\}$ are closed.

Axiom (Independence). For all $f, g, h \in \mathcal{F}$ and $\lambda \in(0,1)$, if $f \succsim g$ then $\lambda f+(1-\lambda) h \succsim$ $\lambda g+(1-\lambda) h$.

Anscombe and Aumann (1963) showed that a binary relation $\succsim$ on $\mathcal{F}$ satisfies these axioms if and only if, for all $f, g \in \mathcal{F}$,

$$
f \succsim g \Leftrightarrow E_{m}(u \circ f) \geq E_{m}(u \circ g),
$$

lotteries involved in these acts. In view of the spurious unanimity issue, it makes sense to restrict (Unambiguous) Pareto Dominance to either common-belief or common-taste acts. The former yields sharper results in the Savage setting adopted by Gilboa, Samet, and Schmeidler (2004) and, similarly, the latter yields sharper results in the Anscombe-Aumann setting used here. 
where $u: X \rightarrow \mathbb{R}$ in non-constant and $m \in \Delta(\mathcal{S})$. ( $u$ is unique up to a positive affine transformation and $m$ is unique.) We then say that $\succsim$ is an $S E U$ preference relation and call $S E U$ representation of $\succsim$ any couple $(u, m)$ satisfying (1).

\section{Preference and unambiguous preference}

Each agent (individual or society) is endowed with a preference relation $\succsim$ on $\mathcal{F}$. We assume that $\succsim$ is an MBA preference relation (for Monotone, Bernoullian, Archimedean), i.e. that it satisfies the axioms of the SEU model except for Independence, which is weakened as follows.

Axiom (Risk Independence). For all $p, q, r \in \mathcal{P}$ and $\lambda \in(0,1)$, if $p \succsim q$ then $\lambda p+(1-\lambda) r \succsim$ $\lambda q+(1-\lambda) r$.

The MBA class thus constrains preferences over lotteries to conform to the Expected Utility model but essentially leaves preferences over uncertain acts unconstrained, except for Monotonicity. Cerreia-Vioglio, Ghirardato, Maccheroni, Marinacci, and Siniscalchi (2011) showed that a binary relation $\succsim$ on $\mathcal{F}$ satisfies these axioms if and only if, for all $f, g \in \mathcal{F}$,

$$
f \succsim g \Leftrightarrow J(u \circ f) \geq J(u \circ g)
$$

where $u: \mathcal{X} \rightarrow \mathbb{R}$ is non-constant and $J: \operatorname{conv}(u(\mathcal{X}))^{\mathcal{S}} \rightarrow \mathbb{R}$ is monotonic, continuous, and normalized. $^{3}$ ( $u$ is unique up to a positive affine transformation, and $J$ is unique given $u$.) We call MBA representation of $\succsim$ any couple $(u, J)$ satisfying (2). Besides the SEU model, many popular ambiguity models fall within the MBA class and correspond to specifications of the MBA functional $J$, such as the Maxmin Expected Utility (henceforth MEU; Gilboa and Schmeidler, 1989), Choquet Expected Utility (henceforth CEU; Schmeidler, 1989), and Smooth Ambiguity (henceforth SA; Klibanoff, Marinacci, and Mukerji, 2005) models.

We also consider a subrelation $\succsim^{*}$ of $\succsim$ on $\mathcal{F}$, which is interpreted as involving the preference comparisons that are unaffected by ambiguity. ${ }^{4} \succsim^{*}$ is called the unambiguous part of $\succsim$, or simply the unambiguous preference relation. We assume that $\succsim^{*}$ is a Bewley preference relation, i.e. that it satisfies the axioms of the SEU model except for Completeness, which is weakened as follows. ${ }^{5}$

Axiom (Risk Completeness). For all $p, q \in \mathcal{P}, p \succsim q$ or $q \succsim p$.

Thus, contrary to $\succsim, ~{ }^{*}$ satisfies Independence but may violate Completeness. The former property captures the interpretation of $\succsim^{*}$ as the unambiguous part of the agent's preferences: since they are unaffected by ambiguity, these preference comparisons cannot be reversed on mixing. The latter property reflects the potential presence of ambiguity in $\succsim$ : there may be preference comparisons that are affected by ambiguity.

\footnotetext{
${ }^{3}$ conv denotes convex hull. That $J$ is monotonic means that for all $f, g \in \mathcal{F}$, if $u \circ f(s) \geq u \circ g(s)$ for all $s \in \mathcal{S}$ then $J(u \circ f) \geq J(u \circ g)$. That $J$ is normalized means that $J(u \circ p)=u \circ p$ for all $p \in \mathcal{P}$.

${ }^{4}$ That $\succsim^{*}$ is a subrelation of $\succsim$ means that $f \succsim^{*} g$ implies $f \succsim g$ for all $f, g \in \mathcal{F}$.

${ }^{5}$ This weakening is usually named "Certainty Completeness".
} 
Bewley (2002) showed that a binary relation $\succsim^{*}$ on $\mathcal{F}$ satisfies these axioms if and only if, for all $f, g \in \mathcal{F}$,

$$
f \succsim^{*} g \Leftrightarrow\left[E_{m}(u \circ f) \geq E_{m}(u \circ g) \text { for all } m \in M\right],
$$

where $u: \mathcal{X} \rightarrow \mathbb{R}$ is non-constant and $M \subseteq \Delta(\mathcal{S})$ is non-empty, compact, and convex. ( $u$ is unique up to a positive affine transformation and $M$ is unique.) (3) states that $f$ is unambiguously preferred to $g$ if and only if $f$ has a higher subjective expected utility than $g$ for all probability distributions in some set $M$, which is then interpreted as capturing the uncertainty perceived by the agent. We shall call $M$ the set of relevant priors of the agent. Clearly, $M$ is a singleton if and only if $\succsim^{*}$ is an SEU preference relation (i.e. if and only if the agent perceives no ambiguity). We call Bewley representation of $\succsim^{*}$ any couple $(u, M)$ satisfying (3).

As is clear from (3), a definition of the unambiguous part of an MBA preference relation $\succsim$ is equivalent to a definition of the relevant priors of this preference relation. The two following definitions of these concepts have been proposed in the literature.

Example 1. Ghirardato, Maccheroni, and Marinacci (2004) and Nehring (2007) define the unambiguous preference relation ${ }^{* \mathrm{GMMN}}$ as follows: for all $f, g \in \mathcal{F}$,

$$
f \succsim^{* \mathrm{GMMN}} g \Leftrightarrow[\lambda f+(1-\lambda) h \succsim \lambda g+(1-\lambda) h \text { for all } h \in \mathcal{F} \text { and } \lambda \in(0,1]]
$$

$\succsim^{* \text { GMMN }}$ deems unambiguous all preferences that cannot be reversed on mixing. It is thus the most complete unambiguous preference that can be defined from $\succsim$ : any subrelation of $\succsim$ satisfying the Bewley axioms is also a subrelation of ${ }^{* \mathrm{GMMN}}$. Equivalently, $\succsim^{* \mathrm{GMMN}}$ yields the smallest possible set of relevant priors, which we denote by $M^{\mathrm{GMMN}}$.

Example 2. Klibanoff, Mukerji, and Seo (2014) consider an MBA preference relation $\succsim$ on the set $\hat{\mathcal{F}}$ of simple acts $\hat{f}: \mathcal{S}^{\infty} \rightarrow \mathcal{P}$, satisfying an "Event Symmetry" axiom stating that the agent views all ordinates of $\mathcal{S}^{\infty}$ as identical, as well as a "Monotone Continuity" axiom. They derive from $\succsim$ a non-empty, closed set $R \subseteq \Delta(\mathcal{S})$ of "relevant measures" as follows: a measure $m \in \Delta(\mathcal{S})$ is deemed relevant if for each neighborhood $M$ of $m$, the event that the limiting frequency over $\mathcal{S}$ lies in $M$ is non-null. The set $M^{\mathrm{KMS}}=\operatorname{conv}(R)$ can be thought of as the set of relevant priors for $\succsim$, and the preference relation generated by it according to (3), call it $\succsim^{* \text { KMS }}$, as the unambiguous part of $\succsim$ (or, at least, as the unambiguous part of its restriction to acts agreeing in all but one ordinate). In general, $\succsim^{* \mathrm{KMS}}$ is less complete than $\succsim^{* \mathrm{GMMN}}$, or equivalently, $M^{\mathrm{GMMN}} \subseteq M^{\mathrm{KMS}}$.

Rather than deriving $\succsim^{*}$ from $\succsim$ as in these examples, one could instead posit $\succsim^{*}$ as an additional primitive relation, in the spirit of Gilboa, Maccheroni, Marinacci, and Schmeidler (2010). ${ }^{6}$ The results in this paper hold no matter the definition retained, as long as $\succsim$ is an MBA preference relation and $\succsim^{*}$ is a Bewley preference subrelation; we summarize this by saying that $\succsim$ is an MBA preference relation with unambiguous part $\succsim^{*}$. Whenever this is the case, $\succsim$ and $\succsim^{*}$ admit the following joint representation. To state it, we say that an act $f \in \mathcal{F}$ is $\succsim^{*}$-crisp if $f \sim^{*} p$ for some $p \in \mathcal{P}$.

\footnotetext{
${ }^{6}$ Note that (Gilboa, Maccheroni, Marinacci, and Schmeidler, 2010) further assume that $\succsim$ is an MEU preference relation, and interpret $\succsim^{*}$ in terms of "objective rationality" rather than unambiguous preference.
} 
Proposition 1 (Cerreia-Vioglio, Ghirardato, Maccheroni, Marinacci, and Siniscalchi, 2011). $\succsim$ is an MBA preference relation on $\mathcal{F}$ with unambiguous part $\succsim^{*}$ if and only if there exist a non-constant function $u: \mathcal{X} \rightarrow \mathbb{R}$, a non-empty, compact, convex set $M \subseteq \Delta(\mathcal{S})$ and a function $\alpha: \mathcal{F} \rightarrow[0,1]$ such that:

- $(u, M)$ is a Bewley representation of $\succsim^{*}$,

- The functional $J: \operatorname{conv}(u(\mathcal{X}))^{\mathcal{S}} \rightarrow \mathbb{R}$ defined by, for all $f \in \mathcal{F}$,

$$
J(u \circ f)=\alpha(f) \min _{m \in M} E_{m}(u \circ f)+(1-\alpha(f)) \max _{m \in M} E_{m}(u \circ f)
$$

is monotonic, continuous, and normalized, and $(u, J)$ is an MBA representation of $\succsim$.

Moreover, $u$ is unique up to a positive affine transformation, $M$ is unique, and $\alpha$ is unique on $\succsim^{*}$-non-crisp acts. ${ }^{7}$

(4) states that $\succsim$ admits a representation generalizing that of Hurwicz (1951): the utility of $f$ is a convex combination of the minimum and maximum subjective expected utilities of $f$ (over the same set $M$ of relevant priors and with the same utility function $u$ as in the representation of $\succsim^{*}$ ), the weight $\alpha(f)$ being interpreted as an index of ambiguity aversion for act $f$. We call generalized Hurwicz representation of $\succsim$ (with unambiguous part $\left.\succsim^{*}\right)$ any triple $(u, M, \alpha)$ satisfying the two properties in Proposition 1.

\section{Pareto Dominance}

We consider a finite set $\mathcal{I}=\{1, \ldots,|\mathcal{I}|\}$ of at least two individuals and let $\mathcal{I}^{\prime}=\{0\} \cup \mathcal{I}$, where 0 stands for society. Each agent $i \in \mathcal{I}^{\prime}$ is endowed with an MBA preference relation $\succsim_{i}$ on $\mathcal{F}$ with unambiguous part $\succsim_{i}^{*}$. In this section we briefly review existing results on aggregation of preferences. This will lay the ground for the new results presented in the next section.

We say that an individual $i \in \mathcal{I}$ is null if there exist no $p, q \in \mathcal{P}$ such that $p \succ_{0} q$ and $p \sim_{j} q$ for all $j \in \mathcal{I} \backslash\{i\}$. We assume that the profile $\left(\succsim_{i}\right)_{i \in \mathcal{I}}$ of individual preference relations satisfies the following axiom.

Axiom (Risk Diversity). For all $i \in \mathcal{I}$, there exist $p, q \in \mathcal{P}$ such that $p \succ_{i} q$ and $p \sim_{j} q$ for all $j \in \mathcal{I} \backslash\{i\}$.

This is a standard axiom in the preference aggregation literature. ${ }^{8}$ For some results we only require the following, weaker axiom. ${ }^{9}$

Axiom (Risk Minimal Agreement). There exist $p, q \in \mathcal{P}$ such that $p \succ_{i} q$ for all $i \in \mathcal{I}$.

\footnotetext{
${ }^{7}$ An act $f$ is $\succsim^{*}$-crisp if and only if the minimum and the maximum agree in (4), in which case the coefficient $\alpha(f)$ is irrelevant. This proposition is a straightforward extension of Cerreia-Vioglio, Ghirardato, Maccheroni, Marinacci, and Siniscalchi (2011)'s result, which focuses on the "only if" part and on the specific unambiguous preference relation $\succsim^{* \text { GMMN }}$ defined above.

${ }^{8}$ It is usually called "Independent Prospects".

${ }^{9}$ To check that this axiom is weaker than Risk Diversity, take two lotteries $p_{i}, q_{i} \in \mathcal{P}$ as in Risk Diversity for each $i \in \mathcal{I}$, and mix them with strictly positive weights.
} 
We shall restrict attention throughout to Pareto criteria involving weak preferences only, but the usual variants involving strict preferences could be straightforwardly accomodated and we omit the details. We start with the standard Pareto Dominance axiom, as well as a restriction of this axiom to the subdomain of lotteries.

Axiom (Pareto Dominance). For all $f, g \in \mathcal{F}$, if $f \succsim_{i} g$ for all $i \in \mathcal{I}$ then $f \succsim_{0} g$.

Axiom (Risk Pareto Dominance). For all $p, q \in \mathcal{P}$, if $p \succsim_{i} q$ for all $i \in \mathcal{I}$ then $p \succsim_{0} q$.

Since MBA preferences satisfy Risk Independence, it is a direct consequence of Harsanyi's aggregation theorem that Risk Pareto Dominance is necessary and sufficient for linear aggregation of taste over outcomes.

Proposition 2 (Harsanyi, 1955). Assume that $\succsim_{i}$ is an MBA preference relation on $\mathcal{F}$ for all $i \in \mathcal{I}^{\prime}$. Then $\left(\succsim_{i}\right)_{i \in \mathcal{I}^{\prime}}$ satisfies Risk Pareto Dominance if and only if, for all MBA representations $\left(u_{i}, J_{i}\right)_{i \in \mathcal{I}^{\prime}}$ of $\left(\succsim_{i}\right)_{i \in \mathcal{I}^{\prime}}$, there exist $\theta \in \mathbb{R}_{+}^{\mathcal{I}} \backslash\{0\}$ and $\gamma \in \mathbb{R}$ such that

$$
u_{0}=\sum_{i \in \mathcal{I}} \theta_{i} u_{i}+\gamma
$$

Moreover, if $\left(\succsim_{i}\right)_{i \in \mathcal{I}}$ satisfies Risk Diversity then $\theta$ and $\gamma$ are unique given $\left(u_{i}\right)_{i \in \mathcal{I}^{\prime}}$ and an individual $i \in \mathcal{I}$ is null if and only if $\theta_{i}=0$.

On the other hand, if $\left(\succsim_{i}\right)_{i \in \mathcal{I}}$ satisfies Risk Diversity then $\left(\succsim_{i}\right)_{i \in \mathcal{I}^{\prime}}$ cannot satisfy Pareto Dominance in general. This was shown by Mongin $(1995,1998)$ within the class of SEU preferences. For such preferences, Pareto Dominance can only be satisfied if $m_{0}=m_{i}$ for all non-null individuals $i \in \mathcal{I}$. ${ }^{10}$ The following example illustrates this impossibility of simultaneously aggregating heterogeneous tastes and beliefs.

Example 3. Let $\mathcal{S}=\left\{s_{1}, s_{2}\right\}, \mathcal{X}=\{x, y, z\}$, and $\mathcal{I}=\{1,2\}$. Assume that $\succsim_{i}$ is an SEU preference relation with SEU representation $\left(u_{i}, m_{i}\right)$ for all $i \in\{0,1,2\}$, where

$$
\begin{array}{ll}
u_{1}(x)=1, u_{1}(y)=0, u_{1}(z)=0, & m_{1}\left(s_{1}\right)=\frac{1}{4}, \\
u_{2}(x)=0, u_{2}(y)=1, u_{2}(z)=0, & m_{2}\left(s_{1}\right)=\frac{3}{4} .
\end{array}
$$

Note that $\left(\succsim_{i}\right)_{i=1,2}$ satisfies Risk Diversity. Then $\left(\succsim_{i}\right)_{i=0,1,2}$ can only satisfy Pareto Dominance if either 1 or 2 is null. Indeed, suppose that $\theta_{1} \theta_{2}>0$ in (5) and, assuming without loss of generality that $\theta_{1}+\theta_{2}=1$, define the acts $f, g \in \mathcal{F}$ by

$$
\begin{aligned}
& f\left(s_{1}\right)=p, f\left(s_{2}\right)=q \text {, } \\
& \text { where } \\
& p=\theta_{2} x+\theta_{1} z, \\
& g\left(s_{1}\right)=q, g\left(s_{2}\right)=p \text {, } \\
& q=\theta_{1} y+\theta_{2} z .
\end{aligned}
$$

Note that $p \succ_{1} q$ and, hence, $g \succ_{1} f$ since $m_{1}\left(s_{2}\right)>m_{1}\left(s_{1}\right)$. Similarly, $q \succ_{2} p$ and, hence, $g \succ_{2} f$ since $m_{2}\left(s_{1}\right)>m_{2}\left(s_{2}\right)$. On the other hand, $p \sim_{0} q$ and, hence, $f \sim_{0} g$ independently of $m_{0}$, so Pareto Dominance is violated. ${ }^{11}$

\footnotetext{
${ }^{10}$ In this common-belief case, any act $f \in \mathcal{F}$ can be identified with the lottery $\sum_{s \in \mathcal{S}} m(s) f(s)$, where $m \in \Delta(\mathcal{S})$ is the common belief, so Pareto Dominance reduces to Risk Pareto Dominance.

${ }^{11}$ Strictly speaking, this only violates the "strict" version of Pareto Dominance. However, it is easy to obtain a violation of the "weak" version by replacing $g$ with $(1-\varepsilon) g+\varepsilon z$ for a small enough $\varepsilon \in(0,1)$. We omit the details here and in subsequent examples.
} 
Mongin $(1995,2014)$ uses the term spurious unanimity to describe situations such as these: individuals unanimously rank $g$ above $f$ but for "different reasons" (opposite differences in tastes and beliefs). Under Risk Diversity, there are always acts inducing spurious unanimity while being socially indifferent, leading to violations of Pareto Dominance. Mongin argues that Pareto Dominance is not compelling when unanimity is spurious.

Gilboa, Samet, and Schmeidler (2004) also argue against Pareto Dominance, specifically in cases where unanimity results from disagreeing beliefs. They propose a restriction of Pareto dominance to "common-belief" acts, and, in a Savage setting, show that it allows aggregation of heterogeneous beliefs.

Moving beyond the SEU class to consider ambiguity-sensitive preferences gives rise to another type of impossibility, extending even to the common-belief case. This was shown by Gajdos, Tallon, and Vergnaud (2008) within the classes of MEU and CEU preferences. For such preferences, Pareto Dominance can only be satisfied if $\succsim_{i}$ is an SEU preference relation for all non-null individuals $i \in \mathcal{I}$, provided there are least two such individuals. ${ }^{12}$ Gajdos, Tallon, and Vergnaud (2008) also provide an example of SA preferences in which the same impossibility arises. The following example illustrates this general impossibility within the class of MEU preferences.

Example 4. Consider again Example 3 but assume now that $\succsim_{i}$ is an MEU preference relation with MEU representation $\left(u_{i}, M_{i}\right)$ for all $i \in\{0,1,2\}$, where $u_{1}$ and $u_{2}$ are as above and

$$
M_{1}=M_{2}=\left\{m \in \Delta(\mathcal{S}): \frac{1}{4} \leq m\left(s_{1}\right) \leq \frac{3}{4}\right\}
$$

Then even though individuals now have the same beliefs, $\left(\succsim_{i}\right)_{i=0,1,2}$ can only satisfy Pareto Dominance if either 1 or 2 is null. Indeed, suppose that $\theta_{1} \theta_{2}>0$ in (5) and consider the act $f$ as above and the lottery $r=\frac{1}{2} p+\frac{1}{2} q \in \mathcal{P}$. Then $r \succ_{1} f$ since $p \succ_{1} q$ and $\min _{m \in M_{1}} m\left(s_{1}\right)<$ $\frac{1}{2}$. Similarly, $r \succ_{2} f$ since $q \succ_{2} p$ and $\min _{m \in M_{2}} m\left(s_{2}\right)<\frac{1}{2}$. On the other hand, $r \sim_{0} f$ independently of $M_{0}$ since $p \sim_{0} q$, so Pareto Dominance is violated.

In line with Mongin's terminology, we refer to situations such as these as spurious hedging: $r=\frac{1}{2} f+\frac{1}{2} g$ is perceived as hedging the ambiguity of $f$ for both individuals but for "different reasons" ( 1 ranks $p$ above $q$ and is therefore concerned with $m\left(s_{1}\right)$ being low when evaluating $f$, and the opposite for 2 ). Moreover, $r$ is not perceived as hedging the ambiguity of $f$ by society. Under Risk Diversity, there are always acts inducing spurious hedging and leading to violations of Pareto Dominance. We may thus argue, along similar lines to those cited above, that Pareto Dominance is less compelling when unanimity results from spurious hedging.

\section{Unambiguous Pareto Dominance}

We show in this section that restricting Pareto Dominance to the unambiguous part of agents' preferences yields a consistent and separate aggregation of beliefs and tastes. This stands in contrast with the impossibilities discussed in the previous section, arising when Pareto Domi-

\footnotetext{
${ }^{12}$ By Mongin's result, these individuals must in addition share the same beliefs. Gajdos, Tallon, and Vergnaud (2008) show more generally that within the wider class of "Rank-Dependent Additive" preferences, these individuals must have "uncertainty-neutral betting preferences".
} 
nance is imposed on the whole preference relation. The restricted Pareto Dominance axiom can be simply stated as follows.

Axiom (Unambiguous Pareto Dominance). For all $f, g \in \mathcal{F}$, if $f \succsim_{i}^{*} g$ for all $i \in \mathcal{I}$ then $f \succsim_{0}^{*} g$.

The axiom states that if all individuals unambiguously prefer $f$ to $g$ then so does society. Since preferences over lotteries are always unambiguous, Unambiguous Pareto Dominance implies Risk Pareto Dominance. On the other hand, Unambiguous Pareto Dominance restricts Pareto Dominance by only constraining social preferences when individual preferences are both unanimous and unambiguous. Intuitively, this restriction makes the axiom robust to the situations of spurious hedging: such situations necessarily involve the ambiguous part of the preference relation, since by definition an unambiguous preference cannot be reversed on mixing (thus in Example 4, we have $r \succ_{i} f$ but not $r \succ_{i}^{*} f$, for $\left.i=1,2\right)$. We obtain the following aggregation result.

Theorem 1. Assume that $\succsim_{i}$ is an MBA preference relation on $\mathcal{F}$ with unambiguous part $\succsim_{i}^{*}$ for all $i \in \mathcal{I}^{\prime}$ and that $\left(\succsim_{i}\right)_{i \in \mathcal{I}}$ satisfies Risk Diversity. Then $\left(\succsim_{i}\right)_{i \in \mathcal{I}^{\prime}}$ satisfies Unambiguous Pareto Dominance if and only if, for all generalized Hurwicz representations $\left(u_{i}, M_{i}, \alpha_{i}\right)_{i \in \mathcal{I}^{\prime}}$ of $\left(\succsim_{i}\right)_{i \in \mathcal{I}^{\prime}}$, there exist $\theta \in \mathbb{R}_{+}^{\mathcal{I}} \backslash\{0\}$ and $\gamma \in \mathbb{R}$ such that (5) holds and

$$
M_{0} \subseteq \bigcap_{i \in \mathcal{I}, \theta_{i}>0} M_{i}
$$

(6) states that each relevant prior for society must also be relevant for each non-null individual. Thus Unambiguous Pareto Dominance allows aggregation of ambiguity-sensitive preferences, even with different sets of relevant priors, as long as they share at least one relevant prior. When this is the case we say that individual beliefs are compatible. If there are several common priors then society can adopt any subset of common priors. In particular, society can have SEU preferences even if all individuals have ambiguity-sensitive preferences. If there is exactly one common prior then society must have SEU preferences with this prior. In particular, any non-null individual with SEU preferences forces society to have SEU preferences with her prior. ${ }^{13}$

Theorem 1 shows that Unambiguous Pareto Dominance is characterized, besides linear aggregation of tastes, by a relationship between individual and social beliefs, independently of ambiguity attitudes. This is intuitive since unambiguous preferences are by definition unaffected by ambiguity attitudes. In the particular case where all individuals have SEU preferences and perceive no ambiguity, we obtain the following generalization of Mongin's result in which society is shown rather than assumed to have SEU preferences. ${ }^{14}$

Corollary 1. Assume that $\succsim_{0}$ is an MBA preference relation on $\mathcal{F}$ with unambiguous part $\succsim_{0}^{*}$, that $\succsim_{i}=\succsim_{i}^{*}$ is an SEU preference relation on $\mathcal{F}$ for each $i \in \mathcal{I}$, and that $\left(\succsim_{i}\right)_{i \in \mathcal{I}}$ satisfies Risk Diversity. Then $\left(\succsim_{i}\right)_{i \in \mathcal{I}^{\prime}}$ satisfies Unambiguous Pareto Dominance if and only if $\succsim_{0}$ is

\footnotetext{
${ }^{13}$ A similar pattern was experimentally observed by Baillon, Cabantous, and Wakker (2012).

${ }^{14}$ This is a generalization of Mongin's result since Unambiguous Pareto Dominance boils down to Pareto Dominance if, beyond the assumptions in the Corollary, we assume that $\succsim_{0}=\succsim_{0}^{*}$ is an SEU preference relation. More generally, Unambiguous Pareto Dominance is equivalent to Pareto Dominance whenever $\succsim_{0}=\succsim_{0}^{* \mathrm{GMMN}}$ (Ghirardato, Maccheroni, and Marinacci, 2004, Proposition 4).
} 
an SEU preference relation and, for all SEU representations $\left(u_{i}, m_{i}\right)_{i \in \mathcal{I}^{\prime}}$ of $\left(\succsim_{i}\right)_{i \in \mathcal{I}^{\prime}}$, there exist $\theta \in \mathbb{R}_{+}^{\mathcal{I}} \backslash\{0\}$ and $\gamma \in \mathbb{R}$ such that (5) holds and $m_{0}=m_{i}$ for all non-null individuals $i \in \mathcal{I}$.

Except in this SEU case, (6) does not force non-null individuals with ambiguity-sensitive preferences to have identical sets of relevant priors. Yet the allowed heterogeneity in beliefs is limited as aggregation is impossible when non-null individuals have incompatible beliefs.

In order to allow aggregation of incompatible beliefs, a further weakening of Unambiguous Pareto Dominance is needed, which makes it robust to spurious unanimity arising from differences of beliefs and tastes. To state it, we say that $f, g \in \mathcal{F}$ are common-taste acts if $p \succsim_{i} q \Leftrightarrow p \succsim_{j} q$ for all $i, j \in \mathcal{I}$ and $p, q \in \operatorname{conv}(f(\mathcal{S}) \cup g(\mathcal{S}))$. Intuitively, common-taste acts are acts involving only lotteries over which all individuals have the same cardinal preferences.

Axiom (Common-Taste Unambiguous Pareto Dominance). For all common-taste acts $f, g \in \mathcal{F}$, if $f \succsim_{i}^{*} g$ for all $i \in \mathcal{I}$ then $f \succsim_{0}^{*} g$.

The axiom states that, for common-taste acts $f$ and $g$, if all individuals unambiguously prefer $f$ to $g$ then so does society. This weakening to common-taste acts does not have any bite in situations of spurious unanimity, since such situations by definition arise from simultaneous differences in tastes and beliefs. Note that Common-Taste Unambiguous Pareto Dominance can alternatively be viewed as the unambiguous restriction of the following axiom.

Axiom (Common-Taste Pareto Dominance). For all common-taste acts $f, g \in \mathcal{F}$, if $f \succsim_{i} g$ for all $i \in \mathcal{I}$ then $f \succsim_{0} g$.

$\mathrm{Qu}$ (2014) recently introduced a weaker version of this latter axiom and characterized it, together with Risk Pareto Dominance, by linear aggregation of beliefs when all agents have SEU preferences. ${ }^{15}$ Our version has the additional strength to imply Risk Pareto Dominance (and so does Common-Taste Unambiguous Pareto Dominance since preferences over lotteries are always unambiguous). ${ }^{16}$ We obtain the following characterization of Common-Taste Unambiguous Pareto Dominance for general MBA preferences.

Theorem 2. Assume that $\succsim_{i}$ is an MBA preference relation on $\mathcal{F}$ with unambiguous part $\succsim_{i}^{*}$ for all $i \in \mathcal{I}^{\prime}$ and that $\left(\succsim_{i}\right)_{i \in \mathcal{I}}$ satisfies Risk Minimal Agreement. Then $\left(\succsim_{i}\right)_{i \in \mathcal{I}^{\prime}}$ satisfies CommonTaste Unambiguous Pareto Dominance if and only if, for all generalized Hurwicz representations $\left(u_{i}, M_{i}, \alpha_{i}\right)_{i \in \mathcal{I}^{\prime}}$ of $\left(\succsim_{i}\right)_{i \in \mathcal{I}^{\prime}}$, there exist $\theta \in \mathbb{R}_{+}^{\mathcal{I}} \backslash\{0\}$ and $\gamma \in \mathbb{R}$ such that (5) holds and

$$
M_{0} \subseteq \operatorname{conv}\left(\bigcup_{i \in \mathcal{I}} M_{i}\right) .
$$

\footnotetext{
${ }^{15} \mathrm{Qu}$ defines common-taste acts more narrowly as acts involving only mixtures of two exogenously given lotteries over which individuals have a unanimous strict preference. His characterization is the same that was obtained by Gilboa, Samet, and Schmeidler (2004) in a Savage setting, by restricting Pareto Dominance to "common-belief" rather than common-taste acts.

${ }^{16}$ Indeed, fix two lotteries $p, q \in \mathcal{P}$ such that $p \succ_{i} q$ for all $i \in \mathcal{I}$. $p$ and $q$ are then common-taste lotteries and, hence, $p \succsim_{0} q$. Moreover, since $\succsim_{0}$ is non-trivial, if $p \sim_{0} q$ then we can find two common-taste lotteries $p^{\prime}, q^{\prime} \in \mathcal{P}$ in the neighborhhod of $p$ and $q$, respectively, such that $p^{\prime} \succ_{i} q^{\prime}$ for all $i \in \mathcal{I}$ and $q^{\prime} \succ_{0} p^{\prime}$, a contradiction. This establishes the strict preference version of Risk Pareto Dominance, which is equivalent to our weak preference version since $\succsim_{0}$ is non-trivial.
} 
(7) states that any socially relevant prior must be a convex combination of some individually relevant priors. The weights in these combinations are not unique in general. ${ }^{17}$

Thus Common-Taste Unambiguous Pareto Dominance allows aggregation of ambiguitysensitive preferences even with incompatible beliefs. As in Theorem 1, society can have SEU preferences even if all individuals have ambiguity-sensitive preferences. The converse is now also possible: society can have ambiguity-sensitive preferences even if all individuals have SEU preferences, in which case social ambiguity results from individual heterogeneity in beliefs. We formally state this corollary, which generalizes Qu's result. ${ }^{18}$

Corollary 2. Assume that $\succsim_{0}$ is an MBA preference relation on $\mathcal{F}$ with unambiguous part $\succsim_{0}^{*}$, that $\succsim_{i}=\succsim_{i}^{*}$ is an SEU preference relation on $\mathcal{F}$ for all $i \in \mathcal{I}$, and that $\left(\succsim_{i}\right)_{i \in \mathcal{I}}$ satisfies Risk Minimal Agreement. Then $\left(\succsim_{i}\right)_{i \in \mathcal{I}^{\prime}}$ satisfies Common-Taste Unambiguous Pareto Dominance if and only if, for all generalized Hurwicz representations $\left(u_{0}, M_{0}, \alpha_{0}\right)$ of $\succsim_{0}$ and SEU representations $\left(u_{i}, m_{i}\right)_{i \in \mathcal{I}}$ of $\left(\succsim_{i}\right)_{i \in \mathcal{I}}$, there exist $\theta \in \mathbb{R}_{+}^{\mathcal{I}} \backslash\{0\}$ and $\gamma \in \mathbb{R}$ such that $(5)$ holds and

$$
M_{0} \subseteq \operatorname{conv}\left(\left\{m_{i}: i \in \mathcal{I}\right\}\right) .
$$

\section{Applications}

Ghirardato, Maccheroni, and Marinacci (2004) , Ghirardato and Siniscalchi (2012), and Klibanoff, Mukerji, and Seo (2014) provide explicit computations of the sets of relevant priors $M^{\mathrm{GMMN}}$ and $M^{\text {KMS }}$ defined in Section 3 for various subclasses of MBA preferences. In this section we use these computations to translate Theorems 1 and 2 in terms of the corresponding representations.

\subsection{MEU preferences}

MEU preferences correspond to MBA functionals of the form

$$
J(u \circ f)=\min _{m \in M} E_{m}(u \circ f),
$$

where $M \subseteq \Delta(\mathcal{S})$ is non-empty, compact and convex. ( $M$ is unique.) We then have

$$
M^{\mathrm{GMMN}}=M^{\mathrm{KMS}}=M
$$

(Ghirardato, Maccheroni, and Marinacci, 2004; Nehring, 2007; Klibanoff, Mukerji, and Seo, 2014). Hence Theorems 1 and 2 translate verbatim in terms of the MEU representations $\left(u_{i}, M_{i}\right)_{i \in \mathcal{I}}$ of $\left(\succsim_{i}\right)_{i \in \mathcal{I}}$ if we let $\succsim_{i}^{*}=\succsim_{i}^{* \mathrm{GMMN}}=\succsim_{i}^{* \mathrm{KMS}}$.

Theorem 1 then stands in contrast with Gajdos, Tallon, and Vergnaud (2008)'s impossibility result: weakening Pareto Dominance to Unambiguous Pareto Dominance allows aggregation of MEU preferences, provided the sets of priors are compatible. Theorem 2, on the other hand,

\footnotetext{
${ }^{17} \mathrm{~A}$ sufficient but very demanding condition for uniqueness of the weights is that all collections $\left(m_{i}, m_{i}^{\prime}\right)_{i \in \mathcal{I}} \in$ $\bigcup_{i \in \mathcal{I}} M_{i}^{2}$ with $m_{i} \neq m_{i}^{\prime}$ for some $i \in \mathcal{I}$ are linearly independent.

${ }^{18}$ This is a generalization of Qu's result since Common-Taste Unambiguous Pareto Dominance boils down to Common-Taste Pareto Dominance if we further assume that $\succsim_{0}=\succsim_{0}^{*}$ is an SEU preference relation. More generally, Common-Taste Unambiguous Pareto Dominance is equivalent to Common-Taste Pareto Dominance whenever $\succsim_{0}=\succsim_{0}^{* G M M N}$ and $\succsim_{0}$ is an MEU or CEU preference relation (see Proposition 5 in the Appendix).
} 
can be compared with an analogous result recently obtained by Qu (2014). Building on results from Crès, Gilboa, and Vieille (2011), he introduces a strengthening of Common-Taste Pareto Dominance and shows that it is characterized by linear aggregation of the sets of priors, i.e.

$$
M_{0}=\left\{\sum_{i \in \mathcal{I}} \lambda_{i} m_{i}:\left(m_{i}\right)_{i \in \mathcal{I}} \in \prod_{i \in \mathcal{I}} M_{i}\right\}
$$

for some $\lambda \in \Delta(\mathcal{I})$. No exact characterization of Common-Taste Pareto Dominance is known. ${ }^{19}$ We first note that for MEU preferences, Common-Taste Pareto Dominance (and, hence, Qu's axiom) is stronger than Common-Taste Unambiguous Pareto Dominance (see Proposition 5 in the Appendix). The following proposition helps assess the additional content of the former with respect to the latter.

Proposition 3. Assume that $\succsim_{i}$ is an MEU preference relation on $\mathcal{F}$ for all $i \in \mathcal{I}^{\prime}$ and that $\left(\succsim_{i}\right)_{i \in \mathcal{I}}$ satisfies Risk Minimal Agreement. If $\left(\succsim_{i}\right)_{i \in \mathcal{I}^{\prime}}$ satisfies Common-Taste Pareto Dominance then, for all MEU representations $\left(u_{i}, M_{i}\right)_{i \in \mathcal{I}^{\prime}}$ of $\left(\succsim_{i}\right)_{i \in \mathcal{I}^{\prime}}$, there exist $\theta \in \mathbb{R}_{+}^{\mathcal{I}} \backslash\{0\}$ and $\gamma \in \mathbb{R}$ such that (5) and (7) hold and for all $\left(m_{i}\right)_{i \in \mathcal{I}} \in \prod_{i \in \mathcal{I}} M_{i}$,

$$
M_{0} \cap \operatorname{conv}\left(\left\{m_{i}: i \in \mathcal{I}\right\}\right) \neq \emptyset .
$$

Thus, Common-Taste Pareto Dominance implies not only that social beliefs must be linear combinations of individual beliefs, but also that any possible selection of individual priors must be aggregated into a social prior (and Qu's axiom further implies that all these selections are aggregated through the same vector of individual weights). This requires the social set of priors to be sufficiently large, and in particular we must have $\bigcap_{i \in \mathcal{I}} M_{i} \subseteq M_{0}$. Hence, for instance, if individuals share more than one prior then society can have SEU preferences under CommonTaste Unambiguous Pareto Dominance but not under Common-Taste Pareto Dominance.

\subsection{CEU preferences}

CEU preferences correspond to MBA functionals of the form

$$
J(u \circ f)=\int_{\mathcal{S}} u \circ f d \nu
$$

where $\nu: 2^{\mathcal{S}} \rightarrow[0,1]$ is a capacity and the integral is taken in the sense of Choquet $(\nu$ is unique). ${ }^{20}$ Equivalently, if we write $\mathcal{S}=\left\{s_{1}, \ldots, s_{N}\right\}$ and define, for each $\sigma \in \operatorname{perm}(N)$, the probability distribution $m_{\nu, \sigma} \in \Delta(\mathcal{S})$ by

$$
m_{\nu, \sigma}\left(s_{n}\right)=\nu\left(\left\{s_{\sigma(1)}, \ldots, s_{\sigma(n)}\right\}\right)-\nu\left(\left\{s_{\sigma(1)}, \ldots, s_{\sigma(n-1)}\right\}\right)
$$

for all $n=1, \ldots, N$, then we have

$$
J(u \circ f)=E_{m_{\nu, \sigma}}(u \circ f)
$$

\footnotetext{
${ }^{19}$ The closest result we are aware of in the literature is Hill (2013, Proposition 2), which, in a different but analogous framework, gives a characterization of Pareto Dominance when the utility functions are the same.

${ }^{20}$ That $\nu$ is a capacity means that $\nu(\emptyset)=0, \nu(\mathcal{S})=1$, and $\nu(S) \leq \nu(T)$ whenever $S \subseteq T$.
} 
for any $\sigma \in \operatorname{perm}(N)$ such that $u \circ f\left(s_{\sigma(1)}\right) \geq \ldots \geq u \circ f\left(s_{\sigma(N)}\right) .{ }^{21}$ We then have

$$
M^{\mathrm{GMMN}}=\operatorname{conv}\left(\left\{m_{\nu, \sigma}: \sigma \in \operatorname{perm}(N)\right\}\right)
$$

(Ghirardato, Maccheroni, and Marinacci, 2004). Thus a decision-maker with a CEU preference relation $\succsim$ with unambiguous part ${ }^{* \text { GMMN }}$ evaluates an act by its subjective expected utility according to a particular element of her set of relevant priors. This element depends on the ranking of states according to her preferences over the lotteries the act yields in each state. The capacity $\nu$ incorporates both perceived ambiguity and ambiguity attitude, in the sense that given any generalized Hurwicz representation $\left(u, M^{\mathrm{GMMN}}, \alpha\right)$ of $\succsim$,

$$
\nu(S)=\alpha(f) \min _{m \in M^{\mathrm{GMMN}}} m(S)+(1-\alpha(f)) \max _{m \in M^{\mathrm{GMMN}}} m(S)
$$

for any event $S \subseteq \mathcal{S}$ and any act $f \in \mathcal{F}$ such that $f(s)=p$ for all $s \in S$ and $f(s)=q$ for all $s \in \mathcal{S} \backslash S$, where $p, q \in \mathcal{P}$ are such that $p \succ q$. We now translate Theorems 1 and 2 in terms of the CEU representations $\left(u_{i}, \nu_{i}\right)_{i \in \mathcal{I}}$ of $\left(\succsim_{i}\right)_{i \in \mathcal{I}}$.

Corollary 3. Assume that $\succsim_{i}$ is a CEU preference relation on $\mathcal{F}$ with unambiguous part $\succsim_{i}^{* \text { GMMN }}$ for all $i \in \mathcal{I}^{\prime}$ and that $\left(\succsim_{i}\right)_{i \in \mathcal{I}}$ satisfies Risk Diversity. Then $\left(\succsim_{i}\right)_{i \in \mathcal{I}^{\prime}}$ satisfies Unambiguous Pareto Dominance if and only if, for all CEU representations $\left(u_{i}, \nu_{i}\right)_{i \in \mathcal{I}^{\prime}}$ of $\left(\succsim_{i}\right)_{i \in \mathcal{I}^{\prime}}$, there exist $\theta \in \mathbb{R}_{+}^{\mathcal{I}} \backslash\{0\}$ and $\gamma \in \mathbb{R}$ such that (5) holds and for all $\sigma \in \operatorname{perm}(N)$,

$$
m_{\nu_{0}, \sigma} \in \bigcap_{i \in \mathcal{I}, \theta_{i}>0} \operatorname{conv}\left(\left\{m_{\nu_{i}, \tau}: \tau \in \operatorname{perm}(N)\right\}\right)
$$

Corollary 4. Assume that $\succsim_{i}$ is a CEU preference relation on $\mathcal{F}$ with unambiguous part $\succsim_{i}^{* \text { GMMN }}$ for all $i \in \mathcal{I}^{\prime}$ and that $\left(\succsim_{i}\right)_{i \in \mathcal{I}}$ satisfies Risk Minimal Agreement. Then $\left(\succsim_{i}\right)_{i \in \mathcal{I}^{\prime}}$ satisfies Common-Taste Unambiguous Pareto Dominance if and only if, for all CEU representations $\left(u_{i}, \nu_{i}\right)_{i \in \mathcal{I}^{\prime}}$ of $\left(\succsim_{i}\right)_{i \in \mathcal{I}^{\prime}}$, there exist $\theta \in \mathbb{R}_{+}^{\mathcal{I}} \backslash\{0\}$ and $\gamma \in \mathbb{R}$ such that (5) holds and for all $\sigma \in \operatorname{perm}(N)$,

$$
m_{\nu_{0}, \sigma} \in \operatorname{conv}\left(\left\{m_{\nu_{i}, \tau}: \tau \in \operatorname{perm}(N), i \in \mathcal{I}\right\}\right) .
$$

Again, Corollary 3 stands in contrast with the Gajdos, Tallon, and Vergnaud (2008)'s impossibility result, and Corollary 4 can be compared with an analogous result recently obtained by $\mathrm{Qu}$ (2014). Indeed, Qu shows that for CEU preferences, his strengthening of Common-Taste Pareto Dominance is characterized by linear aggregation of the capacities, i.e.

$$
\nu_{0}=\sum_{i \in \mathcal{I}} \lambda_{i} \nu_{i}
$$

for some $\lambda \in \Delta(\mathcal{I})$. No exact characterization of Common-Taste Pareto Dominance is known. For CEU preferences, Common-Taste Pareto Dominance (and, hence, Qu's axiom) is again stronger than Common-Taste Unambiguous Pareto Dominance (see Proposition 5 in the Appendix). The following proposition helps assessing the additional content of the former with

\footnotetext{
${ }^{21} \operatorname{perm}(N)$ denotes the set of all permutations of $\{1, \ldots, N\}$.
} 
respect to the latter.

Proposition 4. Assume that $\succsim_{i}$ is a CEU preference relation on $\mathcal{F}$ for all $i \in \mathcal{I}^{\prime}$ and that $\left(\succsim_{i}\right)_{i \in \mathcal{I}}$ satisfies Risk Minimal Agreement. If $\left(\succsim_{i}\right)_{i \in \mathcal{I}^{\prime}}$ satisfies Common-Taste Pareto Dominance then, for all CEU representations $\left(u_{i}, \nu_{i}\right)_{i \in \mathcal{I}^{\prime}}$ of $\left(\succsim_{i}\right)_{i \in \mathcal{I}^{\prime}}$, there exist $\theta \in \mathbb{R}_{+}^{\mathcal{I}} \backslash\{0\}$ and $\gamma \in \mathbb{R}$ such that (5) holds and for all $\sigma \in \operatorname{perm}(N)$,

$$
m_{\nu_{0}, \sigma} \in \operatorname{conv}\left(\left\{m_{\nu_{i}, \sigma}: i \in \mathcal{I}\right\}\right)
$$

(11) strengthens (10) by requiring that the prior used by society to evaluate an act be a linear combination of individual priors corresponding to the same ordering of states. (Note that, since the ordering generated by an act depends on the utility function, it will not in the general be the case that individuals use the priors corresponding to this ordering to evaluate the act in question.) This can be understood to reflect the fact that Common-Taste Pareto Dominance (and Qu's axiom) jointly relates social beliefs and ambiguity attitudes to individual beliefs and ambiguity attitudes. Common-Taste Unambiguous Pareto Dominance, on the other hand, only relates social beliefs to individual beliefs, independently of ambiguity attitudes.

\subsection{SA preferences}

SA preferences correspond to MBA functionals of the form

$$
J(u \circ f)=\phi^{-1}\left(E_{\mu}\left(\phi\left(E_{m}(u \circ f)\right)\right)\right)
$$

where $\phi: \operatorname{conv}(u(\mathcal{X})) \rightarrow \mathbb{R}$ is continuous and strictly increasing and $\mu$ is a countably additive probability measure over $\Delta(\mathcal{S})$. ( $\phi$ is unique up to a positive affine transformation given $u$, and $\mu$ is unique.) We say that a SA representation $(u, \mu, \phi)$ of a SA preference relation $\succsim$ is regular if either $\operatorname{supp}(\mu)$ is finite or there exist $\underline{k}, \bar{k} \in \mathbb{R}_{+} \backslash\{0\}$ such that

$$
\underline{k}|u \circ p-u \circ q| \leq|\phi(u \circ p)-\phi(u \circ q)| \leq \bar{k}|u \circ p-u \circ q|
$$

for all $p, q \in \mathcal{P}$, and that $\succsim$ is regular if it admits such a representation. In this case we have

$$
M^{\mathrm{KMS}}=\operatorname{conv}(\operatorname{supp}(\mu))
$$

(Klibanoff, Mukerji, and Seo, 2014). We now translate Theorems 1 and 2 in terms of the SA representations $\left(u_{i}, \mu_{i}, \phi_{i}\right)_{i \in \mathcal{I}}$ of $\left(\succsim_{i}\right)_{i \in \mathcal{I}}$.

Corollary 5. Assume that $\succsim_{i}$ is a regular SA preference relation on $\mathcal{F}$ with unambiguous part $\succsim_{i}^{* \text { KMS }}$ for all $i \in \mathcal{I}^{\prime}$ and that $\left(\succsim_{i}\right)_{i \in \mathcal{I}}$ satisfies Risk Diversity. Then $\left(\succsim_{i}\right)_{i \in \mathcal{I}^{\prime}}$ satisfies Unambiguous Pareto Dominance if and only if, for all regular SA representations $\left(u_{i}, \mu_{i}, \phi_{i}\right)_{i \in \mathcal{I}^{\prime}}$ of $\left(\succsim_{i}\right)_{i \in \mathcal{I}^{\prime}}$, there exist $\theta \in \mathbb{R}_{+}^{\mathcal{I}} \backslash\{0\}$ and $\gamma \in \mathbb{R}$ such that (5) holds and

$$
\operatorname{supp}\left(\mu_{0}\right) \subseteq \bigcap_{i \in \mathcal{I}, \theta_{i}>0} \operatorname{conv}\left(\operatorname{supp}\left(\mu_{i}\right)\right)
$$


Corollary 6. Assume that $\succsim_{i}$ is a regular SA preference relation on $\mathcal{F}$ with unambiguous part $\succsim_{i}^{* \text { KMS }}$ for all $i \in \mathcal{I}^{\prime}$ and that $\left(\succsim_{i}\right)_{i \in \mathcal{I}}$ satisfies Risk Minimal Agreement. Then $\left(\succsim_{i}\right)_{i \in \mathcal{I}^{\prime}}$ satisfies Common-Taste Unambiguous Pareto Dominance if and only if, for all regular SA representations $\left(u_{i}, \mu_{i}, \phi_{i}\right)_{i \in \mathcal{I}^{\prime}}$ of $\left(\succsim_{i}\right)_{i \in \mathcal{I}^{\prime}}$, there exist $\theta \in \mathbb{R}_{+}^{\mathcal{I}} \backslash\{0\}$ and $\gamma \in \mathbb{R}$ such that (5) holds and

$$
\operatorname{supp}\left(\mu_{0}\right) \subseteq \operatorname{conv}\left(\bigcup_{i \in \mathcal{I}} \operatorname{supp}\left(\mu_{i}\right)\right) .
$$

These are, to our knowledge, the first aggregation results for SA preferences. As Gajdos, Tallon, and Vergnaud (2008)'s counterexample shows, aggregation under Pareto Dominance is impossible in general.

\section{$7 \quad$ Aggregating ambiguity attitudes}

Besides aggregating tastes and beliefs, it may also be of interest to aggregate ambiguity attitudes. That is to say, society may want its reaction to ambiguity to depend on how its individuals react to ambiguity. In this section we simply show that such aggregation is possible, by stating and characterizing an elementary axiom relating individual and social ambiguity attitudes.

Given an MBA preference relation $\succsim$ on $\mathcal{F}$ with unambiguous part $\succsim^{*}$ and an act $f \in \mathcal{F}$, we say that a lottery $p \in \mathcal{P}$ is a lower certainty-equivalent of $f$ if

$$
f \succsim^{*} p \Leftrightarrow \underline{p} \succsim p
$$

for all $p \in \mathcal{P}$, and that a lottery $\bar{p} \in \mathcal{P}$ is an upper certainty-equivalent of $f$ if

$$
p \succsim^{*} f \Leftrightarrow p \succsim \bar{p}
$$

for all $p \in \mathcal{P}$. That is to say, a lower certainty-equivalent of $f$ is a best lottery that is unambiguously worse than $f$, whereas an upper certainty-equivalent of $f$ is a worst lottery that is unambiguously better than $f$. In terms of generalized Hurwicz representation, we have

$$
u \circ \underline{p}=\min _{m \in M} E_{m}(u \circ f), \quad u \circ \bar{p}=\max _{m \in M} E_{m}(u \circ f) .
$$

We therefore have $\bar{p} \succsim f \succsim \underline{p}$, with $\bar{p} \sim f \sim \underline{p}$ if and only if $f$ is $\succsim$-crisp. Thus for an SEU preference relation, lower and upper certainty-equivalents coincide for all acts, whereas for an ambiguity-sensitive preference relation there are always acts for which they do not. For such acts, we then have

$$
f \sim \alpha(f) \underline{p}+(1-\alpha(f)) \bar{p}
$$

Now consider the following axiom.

Axiom (Uncertainy-Adjusted Pareto Dominance). For all $f \in \mathcal{F}$, all lower and upper certainty equivalents $\left(\underline{p}_{i}, \bar{p}_{i}\right)_{i \in \mathcal{I}} \in\left(\mathcal{P}^{2}\right)^{\mathcal{I}^{\prime}}$ of $f$, and all $\lambda \in[0,1]$,

- if $f \succsim_{i} \lambda \underline{p}_{i}+(1-\lambda) \bar{p}_{i}$ for all $i \in \mathcal{I}$ then $f \succsim_{0} \lambda \underline{p}_{0}+(1-\lambda) \bar{p}_{0}$, 
- if $\lambda \underline{p}_{i}+(1-\lambda) \bar{p}_{i} \succsim_{i} f$ for all $i \in \mathcal{I}$ then $\lambda \underline{p}_{0}+(1-\lambda) \bar{p}_{0} \succsim_{0} f$.

The axiom states that if a $\lambda$-mixture of lower and upper certainty-equivalents of $f$ is ranked below (resp. above) $f$ for all individuals then this is also the case for society. Note that the mixing coefficient $\lambda$ is fixed whereas the lower and upper certainty-equivalents are specific to each individual and society. Thus the axiom is a form of Pareto criterion involving an act and a lottery, but the lottery is adjusted for each individual and society in order to account for differences in perceived ambiguity. In view of (12), we immediately obtain the following characterization.

Theorem 3. Assume that $\succsim_{i}$ is an MBA preference relation on $\mathcal{F}$ with unambiguous part $\succsim_{i}^{*}$ for all $i \in \mathcal{I}^{\prime}$. Then $\left(\succsim_{i}\right)_{i \in \mathcal{I}^{\prime}}$ satisfies Uncertainty-Adjusted Pareto Dominance if and only if, for all generalized Hurwicz representations $\left(u_{i}, M_{i}, \alpha_{i}\right)_{i \in \mathcal{I}^{\prime}}$ of $\left(\succsim_{i}\right)_{i \in \mathcal{I}^{\prime}}$ and every $f \in \mathcal{F}$ that is not $\succsim_{0}$-crisp,

$$
\alpha_{0}(f) \in \operatorname{conv}\left(\left\{\alpha_{i}(f): i \in \mathcal{I}, f \text { is not } \succsim_{i^{-}} \text {crisp }\right\}\right)
$$

(13) states that the social ambiguity aversion index for a socially non-crisp act is a convex combination of the ambiguity aversion indices for this act taken over all of the individuals for which the act is not crisp. In other words, society must be less ambiguity averse than the most ambiguity averse individual and more ambiguity averse than the least ambiguity averse (or most ambiguity seeking) individual.

The aggregation of ambiguity attitudes is independent of the aggregation of beliefs and tastes. Thus, for instance, society could disregard individual beliefs while taking into account individual ambiguity attitudes. On the other hand, Theorem 3 can be combined with Theorem 1 or 2 . We state the latter combination as a corollary.

Corollary 7. Assume that $\succsim_{i}$ is an MBA preference relation on $\mathcal{F}$ with unambiguous part $\succsim_{i}^{*}$ for all $i \in \mathcal{I}^{\prime}$ and that $\left(\succsim_{i}\right)_{i \in \mathcal{I}}$ satisfies Risk Minimal Agreement. Then $\left(\succsim_{i}\right)_{i \in \mathcal{I}^{\prime}}$ satisfies Common-Taste Unambiguous Pareto Dominance and Uncertainty-Adjusted Pareto Dominance if and only if, for all generalized Hurwicz representations $\left(u_{i}, M_{i}, \alpha_{i}\right)_{i \in \mathcal{I}^{\prime}}$ of $\left(\succsim_{i}\right)_{i \in \mathcal{I}^{\prime}}$, there exist $\theta \in \mathbb{R}_{+}^{\mathcal{I}} \backslash\{0\}$ and $\gamma \in \mathbb{R}$ such that (5), (7), and (13) hold.

\section{Related literature}

The literature on preference aggregation under expected utility after the pioneering work of Hylland and Zeckhauser (1979), Mongin (1995, 1998) and Gilboa, Samet, and Schmeidler (2004) is relatively scarce. Chambers and Hayashi (2014) and Mongin and Pivato (2013) strengthen the results cited above by replacing the assumption of SEU group preferences by appropriate monotonicity assumptions and, in the former case, a version of Pareto incorporating common knowledge of the appropriate preferences. Billot and Vergopoulos (2014) propose a resolution of the impossibility by defining the society's preferences on "social acts" that embed the individuals' opinions.

There are also relatively few results on aggregation of ambiguity-sensitive preferences in the literature besides Gajdos, Tallon, and Vergnaud (2008)'s impossibility result. Crès, Gilboa, 
and Vieille (2011) showed that within the class of MEU preferences, aggregation under Pareto Dominance is possible if all individuals have the same tastes. As discussed in section 5, Qu (2014) extended this result to the class of CEU preferences and incorporated heterogeneous tastes using a strengthening of Common-Taste Pareto Dominance. Gajdos and Vergnaud (2013), Nascimento (2012) and Hill (2012) give possibility results similar in spirit to that of Crès, Gilboa, and Vieille (2011) for the MEU and Variational preference classes, albeit using different frameworks (information in the form of sets of priors, a richer space of choice objects and a multi-profile setup, respectively).

Compared with these papers, our approach is not restricted to specific models of ambiguity. The MEU and CEU models are quite specific regarding ambiguity attitudes. In the MEU model only ambiguity aversion is possible, not ambiguity seeking. In the CEU model both ambiguity aversion and ambiguity seeking are possible but beliefs and ambiguity attitudes are jointly captured by a capacity; only these capacities are aggregated in Qu's result, not beliefs per se. In contrast, our results apply to a wide range of recent models allowing for more general forms of ambiguity attitudes and yield separate aggregation of beliefs and ambiguity attitudes in all these models. Herzberg (2013) studies aggregation of MBA preferences in a multi-profile setup and gives an extension of Arrow's impossibility theorem to infinite electorates.

Another strand of literature related to the present analysis focuses on the question of how to rank allocations of contingent claims when individuals have heterogeneous beliefs. While using a different conceptual framework and focusing exclusively on SEU maximizers with heterogeneous beliefs, Gilboa, Samuelson, and Schmeidler (2014), Gayer, Gilboa, Samuelson, and Schmeidler (2014), Alon and Gayer (2014), Blume, Cogley, Easley, Sargent, and Tsyrennikov (2014), and Brunnermeier, Simsek, and Xiong (2014) are particularly relevant. These papers propose various restrictions of Pareto Dominance that aim at excluding Pareto improvements that might be based on spurious unanimity arising from differences in beliefs.

Gilboa, Samuelson, and Schmeidler (2014) say that an act $f \in \mathcal{F}$ No-Betting Pareto dominates an act $g \in \mathcal{F}$ if, roughly speaking, $f$ Pareto dominates $g$ and there exists a common belief $m \in \Delta(\mathcal{S})$ such that $E_{m}\left(u_{i} \circ f\right) \geq E_{m}\left(u_{i} \circ g\right)$ for all $i \in \mathcal{I} .{ }^{22}$ It is easy to see that if individuals have compatible beliefs and $f$ Unambiguously Pareto dominates $g$ then $f$ No-Betting Pareto dominates $g$ ( $m$ can then be any member of the intersection of the individuals' sets of relevant priors). If individuals have incompatible beliefs then this implication no longer holds (for instance, if all individuals have SEU preferences then Unambiguous Pareto Dominance reduces to Pareto Dominance, so the implication goes in the other direction). Nevertheless, it is still the case that if $f$ Common-Taste Unambiguously Pareto dominates $g$ then $f$ No-Betting Pareto dominates $g$ ( $m$ can then be any member of the convex hull of the union of the individuals' sets of relevant priors).

Gayer, Gilboa, Samuelson, and Schmeidler (2014) assume that all individuals have SEU preferences and say that an act $f \in \mathcal{F}$ Unanimity Pareto dominates an act $g \in \mathcal{F}$ if, roughly speaking, $E_{m_{j}}\left(u_{i} \circ f\right) \geq E_{m_{j}}\left(u_{i} \circ g\right)$ for all $i, j \in \mathcal{I}$, where $m_{j} \in \Delta(\mathcal{S})$ denotes individual $j$ 's

\footnotetext{
${ }^{22}$ Gilboa, Samuelson, and Schmeidler (2014)'s definition is more sophisticated and involves strict rather than weak preferences. We consider this simple, weak preference version of their definition which is more directly comparable with our axioms.
} 
prior. ${ }^{23}$ It is easy to see that if all individuals have SEU preferences and $f$ Common-Taste Unambiguously Pareto dominates $g$ then $f$ Unanimity Pareto dominates $g$. Alon and Gayer (2014) further assume the society to have MEU preferences and show, in a Savage setting, that the corresponding Unanimity Pareto Dominance axiom is then characterized by (5) and (8). ${ }^{24}$ Unlike Common-Taste Unambiguous Pareto dominance, the very notion of Unanimity Pareto dominance presupposes that all individuals have SEU preferences.

Brunnermeier, Simsek, and Xiong (2014) propose a social welfare function which essentially defines a Bewley social preference relation whose set of priors is the convex hull of the individuals' sets. This can be seen as a particular case of Theorem 2, in which the social set of priors is also allowed to be a strict subset of this convex hull, or equivalently, in which the social preference relation is also allowed to be more complete than the one they propose. Common Taste Unambiguous Pareto Dominance can thus be viewed as an axiomatic basis for (a generalization of) Brunnermeier, Simsek, and Xiong (2014)'s functional.

\section{A Appendix}

\section{A.1 Proof of Theorems 1 and 2}

Since the unambiguous part of an MBA prerference relation is a Bewley preference relation, Theorems 1 and 2 are direct consequences of the following results on aggregation of Bewley preferences, respectively.

Theorem 4. Assume that $\succsim_{i}^{*}$ is a Bewley preference relation on $\mathcal{F}$ for all $i \in \mathcal{I}^{\prime}$ and that $\left(\succsim_{i}^{*}\right)_{i \in \mathcal{I}}$ satisfies Risk Diversity. Then $\left(\succsim_{i}^{*}\right)_{i \in \mathcal{I}^{\prime}}$ satisfies Pareto Dominance if and only if, for all Bewley representations $\left(u_{i}, M_{i}\right)_{i \in \mathcal{I}^{\prime}}$ of $\left(\succsim_{i}^{*}\right)_{i \in \mathcal{I}^{\prime}}$, there exist $\theta \in \mathbb{R}_{+}^{\mathcal{I}} \backslash\{0\}$ and $\gamma \in \mathbb{R}$ such that (5) and (6) hold.

Theorem 5. Assume that $\succsim_{i}^{*}$ is a Bewley preference relation on $\mathcal{F}$ for all $i \in \mathcal{I}^{\prime}$ and that $\left(\succsim_{i}^{*}\right)_{i \in \mathcal{I}}$ satisfies Risk Minimal Agreement. Then $\left(\succsim_{i}^{*}\right)_{i \in \mathcal{I}^{\prime}}$ satisfies Common-Taste Pareto Dominance if and only if, for all Bewley representations $\left(u_{i}, M_{i}\right)_{i \in \mathcal{I}^{\prime}}$ of $\left(\succsim_{i}^{*}\right)_{i \in \mathcal{I}^{\prime}}$, there exist $\theta \in \mathbb{R}_{+}^{\mathcal{I}} \backslash\{0\}$ and $\gamma \in \mathbb{R}$ such that (5) and (7) hold.

We note that these results are also of separate interest since Bewley preferences (and, more generally, incomplete preferences) allow to model either individual indecisiveness or social inability to fully observe individual preferences (Danan, Gajdos, and Tallon, 2013, 2014). In order to prove these results, we first establish an aggregation result for a more general class of incomplete preferences. Define a State-Dependent Expected Multi-Utility preference relation (henceforth SDEMU) on $\mathcal{F}$ as one satisfying the axioms of the SEU model except for Monotonicity, Non-Triviality, and Completeness, the latter being weakened as follows.

Axiom (Reflexivity). For all $f \in \mathcal{F}, f \succsim f$.

\footnotetext{
${ }^{23}$ Again, Gayer, Gilboa, Samuelson, and Schmeidler (2014)'s definition is more sophisticated and involves strict rather than weak preferences.

${ }^{24}$ They rename the axiom Consensus Pareto Dominance.
} 
Note that a Bewley preference relation is an SDEMU preference relation since Risk Completeness and Monotonicity together imply Reflexivity. Nau (2006) and Ok, Ortoleva, and Riella (2012) showed that a binary relation $\succsim^{*}$ on $\mathcal{F}$ satisfies these axioms if and only if, for all $f, g \in \mathcal{F}$,

$$
f \succsim^{*} g \Leftrightarrow\left[\sum_{s \in \mathcal{S}}(w(\cdot, s) \circ f(s)) \geq \sum_{s \in \mathcal{S}}(w(\cdot, s) \circ g(s)) \text { for all } w \in W\right],
$$

where $W \subseteq \mathbb{R}^{\mathcal{X} \times \mathcal{S}}$ is non-empty, closed, and convex. $\left(\operatorname{cl}\left(\operatorname{cone}(W)+\mathbb{R}^{\mathcal{S}}\right)\right.$ is unique. $\left.{ }^{25}\right)$ We call SDEMU representation of $\succsim^{*}$ any $W$ satisfying (14).

Lemma 1. Assume that $\succsim_{i}^{*}$ is an SDEMU preference relation on $\mathcal{F}$ for all $i \in \mathcal{I}^{\prime}$. Then $\left(\succsim_{i}^{*}\right)_{i \in \mathcal{I}^{\prime}}$ satisfies Pareto Dominance if and only if, for all SDEMU representations $\left(W_{i}\right)_{i \in \mathcal{I}^{\prime}}$ of $\left(\succsim_{i}^{*}\right)_{i \in \mathcal{I}^{\prime}}$,

$$
W_{0} \subseteq \operatorname{cl}\left(\sum_{i \in \mathcal{I}} \operatorname{cone}\left(W_{i}\right)+\mathbb{R}^{\mathcal{S}}\right) .
$$

Proof. The proof is a straightforward generalization of the proof of the aggregation theorem in Danan, Gajdos, and Tallon (2014). For all $i \in \mathcal{I}^{\prime}$, the set

$$
K_{i}=\left\{a(f-g): a \in \mathbb{R}_{+}, f, g \in \mathcal{F}, f \succsim_{i}^{*} g\right\}
$$

is a non-empty, closed, convex cone in $\mathbb{R}^{\mathcal{X} \times \mathcal{S}}$, orthogonal to $\mathbb{R}^{\mathcal{S}}$, and a non-empty, closed, convex set $W_{i} \subseteq \mathbb{R}^{\mathcal{X} \times \mathcal{S}}$ is a SDEMU representation of $\succsim_{i}$ if and only if $\operatorname{cl}\left(\operatorname{cone}\left(W_{i}\right)+\mathbb{R}^{\mathcal{S}}\right)=K_{i}^{*}{ }^{26}$ Moreover, $\left(\succsim_{i}^{*}\right)_{i \in \mathcal{I}^{\prime}}$ satisfies Pareto Dominance if and only if $\bigcap_{i \in \mathcal{I}} K_{i} \subseteq K_{0}$, which is equivalent to $K_{0}^{*} \subseteq \operatorname{cl}\left(\sum_{i \in \mathcal{I}} K_{i}^{*}\right)$ (Rockafellar, 1970, Corollary 16.4.2) and, hence, to (15) for all SDEMU representations $\left(W_{i}\right)_{i \in \mathcal{I}^{\prime}}$ of $\left(\succsim_{i}^{*}\right)_{i \in \mathcal{I}^{\prime}}$.

Next, we show that the closure operator in (15) is redundant if $\succsim_{i}^{*}$ is a Bewley preference relation for all $i \in \mathcal{I}$ and $\left(\succsim_{i}^{*}\right)_{i \in \mathcal{I}}$ satisfies Risk Minimal Agreement. Given $u: \mathcal{X} \rightarrow \mathbb{R}$ and $m \in \Delta(\mathcal{S})$, define $w_{u, m}: \mathcal{X} \times \mathcal{S} \rightarrow \mathbb{R}$ by $w_{u, m}(x, s)=m(s) u(x)$. Given $u: \mathcal{X} \rightarrow \mathbb{R}$ and $M \subseteq \Delta(\mathcal{S})$, let $W_{u, M}=\left\{w_{u, m}: m \in M\right\}$. Note that if $\succsim^{*}$ is a Bewley prefrence relation and $(u, M)$ is a Bewley representation of $\succsim^{*}$ then $W_{u, M}$ is a SDEMU representation of $\succsim^{*}$.

Lemma 2. Assume that $\succsim_{i}^{*}$ is a Bewley preference relation on $\mathcal{F}$ for all $i \in \mathcal{I}$ and that $\left(\succsim_{i}^{*}\right)_{i \in \mathcal{I}}$ satisfies Risk Minimal Agreement. Then for all Bewley representations $\left(u_{i}, M_{i}\right)_{i \in \mathcal{I}}$ of $\left(\succsim_{i}^{*}\right)_{i \in \mathcal{I}}$, $\sum_{i \in \mathcal{I}} \operatorname{cone}\left(W_{u_{i}, M_{i}}\right)+\mathbb{R}^{\mathcal{S}}$ is closed.

Proof. By Risk Minimal Agreement, there exist $p, q \in \mathcal{P}$ such taht $p \succ_{i}^{*} q$ for all $i \in \mathcal{I}$. Fix Bewley representations $\left(u_{i}, M_{i}\right)_{i \in \mathcal{I}}$ of $\left(\succsim_{i}^{*}\right)_{i \in \mathcal{I}}$. We first show that cone $\left(W_{u_{i}, M_{i}}\right)+\mathbb{R}^{\mathcal{S}}$ is a closed, convex cone for all $i \in \mathcal{I}$. Convexity is obvious. For closedness, fix $i \in \mathcal{I}$. Then $0 \notin W_{u_{i}, M_{i}}$ since $u_{i}$ is non-constant and, hence, cone $\left(W_{u_{i}, M_{i}}\right)$ is closed since $W_{u_{i}, M_{i}}$ is convex and compact (Rockafellar, 1970, Corollary 9.6.1). Moreover, cone $\left(W_{u_{i}, M_{i}}\right) \cap \mathbb{R}^{\mathcal{S}}=\{0\}$, so cone $\left(W_{u_{i}, M_{i}}\right)+\mathbb{R}^{\mathcal{S}}$ is closed (Rockafellar, 1970, Corollary 9.1.3).

\footnotetext{
${ }^{25}$ cone denotes conical hull. We abuse notation by identifying $\mathbb{R}^{\mathcal{S}}$ with the set of functions $w \in R^{\mathcal{X} \times \mathcal{S}}$ such that $w(\cdot, s)$ is constant for all $s \in \mathcal{S}$.

${ }^{26} K_{i}^{*}$ denotes the dual cone of $K_{i}$, i.e. $K_{i}^{*}=\left\{w \in \mathbb{R}^{\mathcal{X} \times \mathcal{S}}: \sum_{(x, s) \in \mathcal{X} \times \mathcal{S}} w(x, s) k(x, s) \geq 0\right.$ for all $\left.k \in K_{i}\right\}$.
} 
It remains to show that the sum over $\mathcal{I}$ of these closed, convex cones is itself closed. To this end it is sufficient to show that the cones $\left(K_{i}\right)_{i \in \mathcal{I}}$ defined in the proof of Lemma 1 have a common point in their relative interiors (Rockafellar, 1970, Corollary 16.4.2) or, equivalently, that $\left(\succsim_{i}^{*}\right)_{i \in \mathcal{I}}$ satisfies the following property: there exist $f, g \in \mathcal{F}$ such that, for all $i \in \mathcal{I}$ and $g_{i} \in \mathcal{F}$ such that $f \succsim_{i}^{*} g_{i}$, there exist $g_{i}^{\prime} \in \mathcal{F}$ and $\lambda_{i} \in(0,1)$ such that $f \succsim_{i}^{*} g_{i}^{\prime}$ and $g=\lambda_{i} g_{i}+\left(1-\lambda_{i}\right) g_{i}^{\prime}$ (Danan, Gajdos, and Tallon, 2014).

To establish this property, recall that $p \succ_{i}^{*} q$ for all $i \in \mathcal{I}$. By Mixture Continuity and since $\mathcal{X}$ is finite, for all $i \in \mathcal{I}$, there exists an open neighborhood $P_{i}$ of $q$ in $\mathcal{P}$ such that $p \succ_{i}^{*} q^{\prime}$ for all $q^{\prime} \in P_{i}$. Let $P=\bigcap_{i \in \mathcal{I}} P_{i}$, so that $p \succ_{i}^{*} q^{\prime}$ for all $q^{\prime} \in P$ and $i \in \mathcal{I}$. Since $\mathcal{I}$ is finite, there exists a lottery $r \in P \cap \operatorname{int}(\mathcal{P}) .{ }^{27}$ Now, fix $i \in \mathcal{I}$ and $g_{i} \in \mathcal{F}$ such that $p \succsim_{i}^{*} g_{i}$. Given $\lambda \in(0,1)$, let $g_{i}^{\prime}=\frac{1}{1-\lambda} r-\frac{\lambda}{1-\lambda} g$, so that $r=\lambda g_{i}+(1-\lambda) g_{i}^{\prime}$. Since $\mathcal{S}$ is finite, there exists $\lambda \in(0,1)$ small enough so that $g_{i}^{\prime} \in P^{\mathcal{S}} \subset \mathcal{F}$ and, hence, $p \succ_{i}^{*} g_{i}^{\prime}(s)$ for all $s \in \mathcal{S}$. By Monotonicity, it follows that $p \succsim_{i}^{*} g^{\prime}$.

We are now ready to prove Theorems 4 and 5 .

Proof of Theorem 4. Obviously, (5) and (6) imply that $\left(\succsim_{i}^{*}\right)_{i \in \mathcal{I}^{\prime}}$ satisfies Pareto Dominance. Conversely, assume $\left(\succsim_{i}^{*}\right)_{i \in \mathcal{I}^{\prime}}$ satisfies Pareto Dominance. Fix Bewley representations $\left(u_{i}, M_{i}\right)_{i \in \mathcal{I}^{\prime}}$ of $\left(\succsim_{i}^{*}\right)_{i \in \mathcal{I}^{\prime}}$. First, restricting attention to lotteries and using Risk Pareto Dominance and Risk Diversity, we apply Proposition 2 to obtain unique $\theta \in \mathbb{R}_{+}^{\mathcal{I}} \backslash\{0\}$ and $\gamma \in \mathbb{R}$ such that (5) holds. Second, fix $m_{0} \in M_{0}$. In order to establish (6), it is sufficient to show that $m_{0} \in M_{i}$ for all $i \in \mathcal{I}$ such that $\theta_{i}>0$. To this end, note that by Lemmas 1 and 2 , there exist $\left(m_{i}\right)_{i \in \mathcal{I}} \in \prod_{i \in \mathcal{I}} M_{i}$, $\theta^{\prime} \in \mathbb{R}_{+}^{\mathcal{I}}$, and $c \in \mathbb{R}^{\mathcal{S}}$ such that

$$
w_{u_{0}, m_{0}}=\sum_{i \in \mathcal{I}} \theta_{i}^{\prime} w_{u_{i}, m_{i}}+c
$$

and, hence,

$$
m_{0}(s) u_{0}(x)=\sum_{i \in I} \theta_{i}^{\prime} m_{i}(s) u_{i}(x)+c(s)
$$

for all $s \in \mathcal{S}$ and $x \in \mathcal{X}$. Summing over $\mathcal{S}$ yields

$$
u_{0}(x)=\sum_{i \in I} \theta_{i}^{\prime} u_{i}(x)+\sum_{s \in \mathcal{S}} c(s)
$$

for all $x \in \mathcal{X}$, so that $\theta=\theta^{\prime}$ and $\gamma=\sum_{s \in \mathcal{S}} c(s)$. Hence (16) implies that

$$
m_{0}(s)\left(u_{0} \circ p-u_{0} \circ q\right)=\sum_{i \in \mathcal{I}} \theta_{i} m_{i}(s)\left(u_{i} \circ p-u_{i} \circ q\right)
$$

and, hence, using (5), that

$$
\sum_{i \in \mathcal{I}} \theta_{i}\left(m_{0}(s)-m_{i}(s)\right)\left(u_{i} \circ p-u_{i} \circ q\right)=0
$$

\footnotetext{
${ }^{27}$ int denotes interior.
} 
for all $s \in \mathcal{S}$ and $p, q \in \mathcal{P}$. Now, fix $i \in \mathcal{I}$ such that $\theta_{i}>0$. By Risk Diversity, there exist $p, q \in \mathcal{P}$ such that $u_{i} \circ p>u_{i} \circ q$ and $u_{j} \circ p=u_{j} \circ q$ for all $j \in \mathcal{I} \backslash\{i\}$. By (17), it follows that $m_{0}(s)=m_{i}(s)$ for all $s \in \mathcal{S}$, so that $m_{0}=m_{i} \in M_{i}$.

Proof of Theorem 5. Obviously, (5) and (7) imply that $\left(\succsim_{i}^{*}\right)_{i \in \mathcal{I}^{\prime}}$ satisfies Common-Taste Pareto Dominance. Conversely, assume $\left(\succsim_{i}^{*}\right)_{i \in \mathcal{I}^{\prime}}$ satisfies Common-Taste Pareto Dominance. Fix Bewley representations $\left(u_{i}, M_{i}\right)_{i \in \mathcal{I}^{\prime}}$ of $\left(\succsim_{i}^{*}\right)_{i \in \mathcal{I}^{\prime}}$. First, restricting attention to lotteries and using Risk Pareto Dominance, we apply Proposition 2 to obtain $\theta \in \mathbb{R}_{+}^{\mathcal{I}} \backslash\{0\}$ and $\gamma \in \mathbb{R}$ such that (5) holds. Second, by Risk Minimal Agreement, there exist $p, q \in \mathcal{P}$ such that $p \succ_{i}^{*} q$ for all $i \in \mathcal{I}$. Hence all acts in $\operatorname{conv}(\{p, q\})^{\mathcal{S}}$ are common-taste acts. It follows that $p \succ_{0}^{*} q$ by (5), so that individual and social preferences all agree on $\operatorname{conv}(\{p, q\})$. Hence for all $i \in \mathcal{I}$, there exist $a_{i} \in \mathbb{R}_{++}$and $b_{i} \in \mathbb{R}$ such that

$$
u_{i} \circ r=\left(a_{i} u_{0}+b_{i}\right) \circ r
$$

for all $r \in \operatorname{conv}(\{p, q\})$. We can therefore use Common-Taste Pareto Dominance to show, as in the proof of Theorem 4 , that for all $m_{0} \in M_{0}$, there exist $\left(m_{i}\right)_{i \in \mathcal{I}} \in \prod_{i \in \mathcal{I}} M_{i}, \theta^{\prime} \in \mathbb{R}_{+}^{\mathcal{I}}$, and $c^{\prime} \in \mathbb{R}^{\mathcal{S}}$ such that

$$
m_{0}(s) u_{0} \circ r=\sum_{i \in \mathcal{I}} \theta_{i}^{\prime} m_{i}(s) u_{i} \circ r+c^{\prime}(s)
$$

for all $s \in \mathcal{S}$ and $r \in \operatorname{conv}(\{p, q\})$. Summing over $\mathcal{S}$ and using (18) yields

$$
u_{0} \circ r=\sum_{i \in \mathcal{I}} \theta_{i}^{\prime} u_{i} \circ r+\sum_{s \in \mathcal{S}} c^{\prime}(s)=\sum_{i \in \mathcal{I}} \theta_{i}^{\prime} a_{i} u_{0} \circ r+\sum_{i \in \mathcal{I}} \theta_{i}^{\prime} b_{i}+\sum_{s \in \mathcal{S}} c^{\prime}(s)
$$

for all $r \in \operatorname{conv}(\{p, q\})$, so that $\sum_{i \in \mathcal{I}} \theta_{i}^{\prime} a_{i}=1$ and $\sum_{i \in \mathcal{I}} \theta_{i}^{\prime} b_{i}=-\sum_{s \in \mathcal{S}} c^{\prime}(s)$ since $u_{0}$ is nonconstant on $\operatorname{conv}(\{p, q\})$. Hence (19) implies that

$$
m_{0}(s)\left(u_{0} \circ p-u_{0} \circ q\right)=\sum_{i \in \mathcal{I}} \theta_{i}^{\prime} m_{i}(s)\left(u_{i} \circ p-u_{i} \circ q\right)=\sum_{i \in \mathcal{I}} \theta_{i}^{\prime} m_{i}(s) a_{i}\left(u_{0} \circ p-u_{0} \circ q\right)
$$

and, hence, that

$$
m_{0}(s)=\sum_{i \in \mathcal{I}} \theta_{i}^{\prime} a_{i} m_{i}(s)
$$

for all $s \in \mathcal{S}$, so that $m_{0}=\sum_{i \in \mathcal{I}} \theta_{i}^{\prime} a_{i} m_{i}$. Let $\lambda=\left(\theta_{i}^{\prime} a_{i}\right)_{i \in \mathcal{I}} \in \mathbb{R}^{\mathcal{I}}$. Since $\theta_{i}^{\prime} \geq 0$ and $a_{i}>0$ for all $i \in \mathcal{I}$ and $\sum_{i \in \mathcal{I}} \theta_{i}^{\prime} a_{i}=1$, we have $\lambda \in \Delta(\mathcal{I})$ and, hence, $m_{0} \in \operatorname{conv}\left(\left\{m_{i}: i \in \mathcal{I}\right\}\right)$, establishing (7).

\section{A.2 Proof of Propositions 3 and 4}

We first show that if $\succsim_{i}$ is an MEU or CEU preference relation on $\mathcal{F}$ with unambiguous part $\succsim_{i}^{* \text { GMMN }}$ for all $i \in \mathcal{I}^{\prime}$, then Common-Taste Pareto Dominance implies Common-Taste Unambiguous Pareto Dominance. More generally, Ghirardato, Maccheroni, and Marinacci (2004) 
define an Invariant Biseparable preference relation on $\mathcal{F}$ as one satisfying the axioms of the SEU model except for Independence, which is weakened to the "Certainty Independence" axiom of Gilboa and Schmeidler (1989). The Invariant Biseparable class is contained in the MBA class and contains the MEU and CEU classes.

Proposition 5. Assume that $\succsim_{i}$ is an Invariant Biseparable preference relation on $\mathcal{F}$ with unambiguous part $\succsim_{i}^{* \mathrm{GMMN}}$ for all $i \in \mathcal{I}^{\prime}$. If $\left(\succsim_{i}\right)_{i \in \mathcal{I}^{\prime}}$ satisfies Common-Taste Pareto Dominance then $\left(\succsim_{i}\right)_{i \in \mathcal{I}^{\prime}}$ satisfies Common-Taste Unambiguous Pareto Dominance.

Proof. Assume $\left(\succsim_{i}\right)_{i \in \mathcal{I}^{\prime}}$ satisfies Common-Taste Pareto Dominance. Fix two common-taste acts $f, g \in \mathcal{F}$ and let $P=\operatorname{conv}(f(\mathcal{S}) \cup g(\mathcal{S}))$. Given a binary relation $\succsim$ on $\mathcal{F}$, let $\left.\succsim\right|_{P}$ denote its restriction to $P^{\mathcal{S}}$. Clearly, $\left(\left.\succsim_{i}\right|_{P}\right)_{i \in \mathcal{I}^{\prime}}$ satisfies Pareto Dominance and, hence, Unambiguous Pareto Dominance by Ghirardato, Maccheroni, and Marinacci (2004, Proposition 4), i.e. $\left(\left.\succsim_{i}\right|_{P} ^{* \text { GMMN }}\right)_{i \in \mathcal{I}^{\prime}}$ satisfies Pareto Dominance. We want to show that $\left(\succsim_{i}\right)_{i \in \mathcal{I}^{\prime}}$ satisfies CommonTaste Unambiguous Pareto Dominance, i.e. that $\left(\left.\succsim_{i}^{* \mathrm{GMMN}}\right|_{P}\right)_{i \in \mathcal{I}^{\prime}}$ satisfies Pareto Dominance. To this end it is sufficient to show that $\left.\succsim\right|_{P} ^{* G M M N}=\left.\succsim^{* \mathrm{GMMN}}\right|_{P}$ for any Invariant Biseparable preference relation $\succsim$ on $\mathcal{F}$.

If $\left.\succsim\right|_{P}$ is trivial then the result directly follows from Monotonicity, so suppose it is not. By Ghirardato, Maccheroni, and Marinacci (2004, Lemma 1), there then exists a non-constant function $u: \mathcal{X} \rightarrow \mathbb{R}$ and a monotonic, constant-linear functional $J^{\prime}: \mathbb{R}^{\mathcal{S}} \rightarrow \mathbb{R}$ such that

$$
f^{\prime} \succsim g^{\prime} \Leftrightarrow J^{\prime}\left(u \circ f^{\prime}\right) \geq J^{\prime}\left(u \circ g^{\prime}\right)
$$

for all $f^{\prime}, g^{\prime} \in \mathcal{F}$. Moreover, $J^{\prime}$ is unique (independently of $u$ ). Hence $J^{\prime}$ is also the unique such functional such that

$$
\left.f^{\prime} \succsim\right|_{P} g^{\prime} \Leftrightarrow J^{\prime}\left(u \circ f^{\prime}\right) \geq J^{\prime}\left(u \circ g^{\prime}\right)
$$

for all $f^{\prime}, g^{\prime} \in P^{\mathcal{S}}$. By (Ghirardato, Maccheroni, and Marinacci, 2004, Theorem 14), it follows that $\succsim$ and $\left.\succsim\right|_{P}$ induce the same set of relevant priors (namely the Clarke differential of $J^{\prime}$ at $0)$. Hence $\succsim_{P}^{* \mathrm{GMMN}}=\left.\succsim^{* \mathrm{GMMN}}\right|_{P}$ by $(3)$.

Proof of Proposition 3. Assume $\left(\succsim_{i}\right)_{i \in \mathcal{I}^{\prime}}$ satisfies Common-Taste Pareto Dominance. Fix MEU representations $\left(u_{i}, M_{i}\right)_{i \in \mathcal{I}^{\prime}}$ of $\left(\succsim_{i}\right)_{i \in \mathcal{I}^{\prime}}$. Then $(5)$ and $(7)$ hold by Proposition 5 and Theorem 2. We prove (9) by contradiction.

Suppose there exists $\left(m_{i}\right)_{i \in \mathcal{I}} \in \prod_{i \in \mathcal{I}} M_{i}$ such that $M_{0} \cap \operatorname{conv}\left(\left\{m_{i}: i \in \mathcal{I}\right\}\right)=\emptyset$. There then exist $c \in \mathbb{R}^{\mathcal{S}}$ and $d \in \mathbb{R}$ such that $\sum_{s \in \mathcal{S}} c(s) m(s)>d$ for all $m \in M_{0}$ and $\sum_{s \in \mathcal{S}} c(s) m_{i}(s) \leq d$ for all $i \in \mathcal{I}$. We can assume without loss of generality that $|c(s)| \leq \frac{1}{2}$ for all $s \in \mathcal{S}$ and $|d| \leq \frac{1}{2}$. Moreover, by Risk Minimal Agreement, there exist $p, q \in \mathcal{P}$ such that $p \succ_{i} q$ for all $i \in \mathcal{I}$ and, hence, $p \succ_{0} q$ by Common-Taste Pareto Dominance. Define $r \in \mathcal{P}$ and $f \in \mathcal{F}$ by

$$
r=\left(\frac{1}{2}+d\right) p+\left(\frac{1}{2}-d\right) q, \quad f(s)=\left(\frac{1}{2}+c(s)\right) p+\left(\frac{1}{2}-c(s)\right) q
$$

for all $s \in \mathcal{S}$. Note that $f$ and $r$ are common-taste acts. Moreover, for all $m \in \Delta(\mathcal{S})$ and 
$u \in \mathbb{R}^{\mathcal{X}}$

$$
E_{m}(u \circ f)-u \circ r=\left(\sum_{s \in \mathcal{S}} c(s) m(s)-d\right)(u \circ p-u \circ q) .
$$

Hence

$$
\min _{m \in M_{i}} E_{m}\left(u_{i} \circ f\right)-\min _{m \in M_{i}} E_{m}\left(u_{i} \circ r\right)=\min _{m \in M_{i}} E_{m}\left(u_{i} \circ f\right)-u_{i} \circ r \leq E_{m_{i}}\left(u_{i} \circ f\right)-u_{i} \circ r \leq 0
$$

for all $i \in \mathcal{I}$, whereas

$$
\min _{m \in M_{0}} E_{m}\left(u_{0} \circ f\right)-\min _{m \in M_{0}} E_{m}\left(u_{0} \circ r\right)=\min _{m \in M_{0}} E_{m}\left(u_{0} \circ f\right)-u_{0} \circ r>0
$$

since $M_{0}$ is compact. Hence $r \succsim_{i} f$ for all $i \in \mathcal{I}$ and $f \succ_{0} r$, contradicting Common-Taste Pareto Dominance.

Proof of Proposition 4. Assume $\left(\succsim_{i}\right)_{i \in \mathcal{I}^{\prime}}$ satisfies Common-Taste Pareto Dominance. Fix CEU representations $\left(u_{i}, \nu_{i}\right)_{i \in \mathcal{I}^{\prime}}$ of $\left(\succsim_{i}\right)_{i \in \mathcal{I}^{\prime}}$. Then (5) holds by Proposition 5 and Theorem 2. We prove (11) by contradiction.

Suppose there exists $\sigma \in \operatorname{perm}(N)$ such that $m_{\nu_{0}, \sigma} \notin \operatorname{conv}\left(\left\{m_{\nu_{i}, \sigma}: i \in \mathcal{I}\right\}\right)$. There then exist $c \in \mathbb{R}^{\mathcal{S}}$ and $d \in \mathbb{R}$ such that $\sum_{s \in \mathcal{S}} c(s) m_{\nu_{0}, \sigma}(s)>d$ and $\sum_{s \in \mathcal{S}} c(s) m_{\nu_{i}, \sigma}(s) \leq d$ for all $i \in \mathcal{I}$. We can assume without loss of generality that $|c(s)| \leq \frac{1}{N+1}$ for all $s \in \mathcal{S}$ and $|d| \leq \frac{1}{N+1}$. By Risk Minimal Agreement, there exist $p, q \in \mathcal{P}$ such that $p \succ_{i} q$ for all $i \in \mathcal{I}$ and, hence, $p \succ_{0} q$ by Common-Taste Pareto Dominance. Define $f, g \in \mathcal{F}$ by

$$
\begin{aligned}
& f\left(s_{\sigma(n)}\right)=\left(\frac{N+1-n}{N+1}+d\right) p+\left(\frac{n}{N+1}-d\right) q, \\
& g\left(s_{\sigma(n)}\right)=\left(\frac{N+1-n}{N+1}+c(s)\right) p+\left(\frac{n}{N+1}-c(s)\right) q,
\end{aligned}
$$

for all $n=1 \ldots N$. Note that $f$ and $g$ are common-taste acts and that $u_{i} \circ f\left(s_{\sigma(1)}\right) \geq \ldots \geq$ $u_{i} \circ f\left(s_{\sigma(N)}\right)$ and $u_{i} \circ g\left(s_{\sigma(1)}\right) \geq \ldots \geq u_{i} \circ g\left(s_{\sigma(N)}\right)$ for all $i \in \mathcal{I}^{\prime}$. Hence

$$
\begin{aligned}
\int_{\mathcal{S}} u_{i} \circ g d \nu-\int_{\mathcal{S}} u_{i} \circ f d \nu & =E_{m_{\nu_{i}, \sigma}}(u \circ g)-E_{m_{\nu_{i}, \sigma}}(u \circ f) \\
& =\left(\sum_{s \in \mathcal{S}} c(s) m(s)-d\right)\left(u_{i} \circ p-u_{i} \circ q\right)
\end{aligned}
$$

for all $i \in \mathcal{I}^{\prime}$. Hence $f \succsim_{i} g$ for all $i \in \mathcal{I}$ and $g \succ_{0} f$, contradicting Common-Taste Pareto Dominance.

\section{References}

Alon, S., And G. Gayer (2014): "Utilitarian Preferences with Multiple Priors," Mimeo.

Anscombe, F. J., and R. J. Aumann (1963): "A Definition of Subjective Probability," The Annals of Mathematical Statistics, 34(1), 199-205. 
Baillon, A., L. Cabantous, and P. P. Wakker (2012): "Aggregating imprecise or conflicting beliefs: An experimental investigation using modern ambiguity theories," Journal of Risk and Uncertainty, 44(2), 115-147.

Bewley, T. F. (2002): "Knightian decision theory. Part I," Decisions in Economics and Finance, 25(2), 79-110.

Billot, A., And V. Vergopoulos (2014): "Utilitarianism with Prior Heterogeneity," Mimeo.

Blume, L. E., T. Cogley, D. A. Easley, T. J. Sargent, and V. Tsyrennikov (2014): "The Case for Incomplete Markets," Mimeo.

Brunnermeier, M. K., A. Simsek, and W. Xiong (2014): "A Welfare Criterion for Models with Distorted Beliefs," The Quarterly Journal of Economics, forthcoming.

Cerreia-Vioglio, S., P. Ghirardato, F. Maccheroni, M. Marinacci, and M. SinisCALCHI (2011): "Rational preferences under ambiguity," Economic Theory, 48(2-3), 341-375.

Chambers, C. P., and T. Hayashi (2014): "Preference aggregation with incomplete information," Econometrica, 82(2), 589-599.

Crès, H., I. GilboA, And N. Vieille (2011): "Aggregation of multiple prior opinions," Journal of Economic Theory, 146(6), 2563-2582.

Danan, E., T. Gajdos, and J.-M. Tallon (2013): "Aggregating sets of von NeumannMorgenstern utilities," Journal of Economic Theory, 148(2), 663-688.

(2014): "Harsanyi's Aggregation Theorem with Incomplete Preferences," American Economic Journal: Microeconomics, forthcoming.

Ellsberg, D. (1961): "Risk, Ambiguity, and the Savage Axioms," The Quarterly Journal of Economics, 75(4), 643-669.

Gajdos, T., J.-M. Tallon, and J.-C. Vergnaud (2008): "Representation and aggregation of preferences under uncertainty," Journal of Economic Theory, 141(1), 68-99.

Gajdos, T., And J.-C. Vergnaud (2013): "Decisions with conflicting and imprecise information," Social Choice and Welfare, 41(2), 427-452.

Gayer, G., I. Gilboa, L. Samuelson, and D. Schmeidler (2014): "Pareto Efficiency with Different Beliefs," Mimeo.

Ghirardato, P., F. Maccheroni, and M. Marinacci (2004): "Differentiating ambiguity and ambiguity attitude," Journal of Economic Theory, 118(2), 133-173.

Ghirardato, P., and M. Siniscalchi (2012): "Ambiguity in the Small and in the Large," Econometrica, 80(6), 2827-2847.

Gilboa, I., F. Maccheroni, M. Marinacci, and D. Schmeidler (2010): "Objective and Subjective Rationality in a Multiple Prior Model," Econometrica, 78(2), 755-770. 
Gilboa, I., D. Samet, and D. Schmeidler (2004): "Utilitarian Aggregation of Beliefs and Tastes," Journal of Political Economy, 112(4), pp. 932-938.

Gilboa, I., L. Samuelson, and D. Schmeidler (2014): "No-Betting-Pareto Dominance," Econometrica, forthcoming.

Gilboa, I., And D. Schmeidler (1989): "Maxmin expected utility with non-unique prior," Journal of Mathematical Economics, 18(2), 141-153.

HaRsanyi, J. C. (1955): "Cardinal Welfare, Individualistic Ethics, and Interpersonal Comparisons of Utility," Journal of Political Economy, 63(4), 309-321.

HerzberG, F. (2013): "Aggregation of Monotonic Bernoullian Archimedean preferences: Arrovian impossibility results," Discussion Paper W.P. 488, Center for Mathematical Economics, Bielefeld.

HILl, B. (2012): "Unanimity and the aggregation of multiple prior opinions," Mimeo.

(2013): "Dynamic Consistency and Ambiguity: A Reappraisal," HEC Paris Research Paper No ECO/SCD-2013-983.

Hurwicz, L. (1951): "Optimality Criteria for Decision Making under Ignorance," Discussion Paper 370, Cowles Commission Discussion Papers: Statistics.

Hylland, A., and R. Zeckhauser (1979): "The Impossibility of Bayesian Group Decision Making with Separate Aggregation of Beliefs and Values," Econometrica, 47(6), 1321-1336.

Klibanoff, P., M. Marinacci, and S. Mukerji (2005): "A Smooth Model of Decision Making under Ambiguity," Econometrica, 73(6), 1849-1892.

Klibanoff, P., S. Mukerji, and K. Seo (2014): "Perceived Ambiguity and Relevant Measures," Econometrica, forthcoming.

Mongin, P. (1995): "Consistent Bayesian Aggregation," Journal of Economic Theory, 66(2), 313-351.

(1998): "The paradox of the Bayesian experts and state-dependent utility theory," Journal of Mathematical Economics, 29(3), 331-361.

(2014): "Spurious unanimity and the Pareto Principle," Economics and Philosophy, forthcoming.

Mongin, P., And M. Pivato (2013): "Ranking Multidimensional Alternatives and Uncertain Prospects," Mimeo.

NAscimento, L. (2012): "The ex ante aggregation of opinions under uncertainty," Theoretical Economics, 7(3), 535-570.

NAU, R. (2006): "The shape of incomplete preferences," The Annals of Statistics, 34(5), 24302448 . 
Nehring, K. (2007): "Bernoulli Without Bayes: A Theory of Utility-Sophisticated Preferences under Ambiguity," Mimeo.

Ok, E. A., P. Ortoleva, and G. Riella (2012): "Incomplete Preferences Under Uncertainty: Indecisiveness in Beliefs versus Tastes," Econometrica, 80(4), 1791-1808.

QU, X. (2014): "Separate Aggregation of Beliefs and Values under Ambiguity," Mimeo.

Rockafellar, R. T. (1970): Convex analysis. Princeton University Press.

Savage, L. J. (1954): The Foundations of Statistics. John Wiley and Sons.

SchmeIdleR, D. (1989): "Subjective Probability and Expected Utility without Additivity," Econometrica, 57(3), 571-587. 